\title{
A comparison between differently skilled pre-hospital emergency care providers in major incident triage in South Africa
}

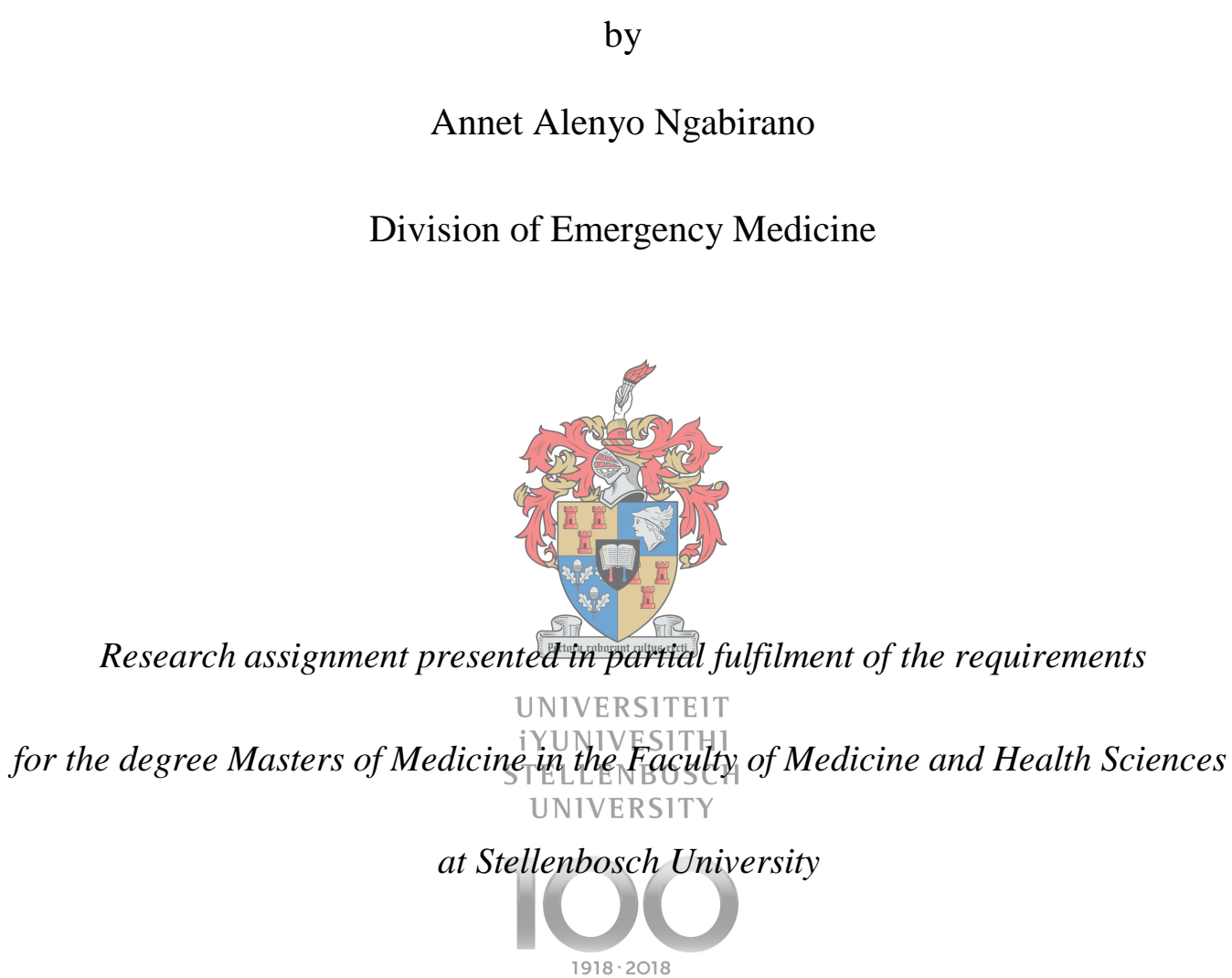

$1918 \cdot 2018$

Supervisors:

Dr. Daniël Jacobus van Hoving (University of Stellenbosch)

Dr. Wayne Patrick Smith (University of Cape Town)

December 2018 


\section{Declaration}

By submitting this dissertation electronically, I, Annet Alenyo Ngabirano declare that the entirety of the work contained therein is my own, original work, that I am the sole author thereof (save to the extent explicitly otherwise stated), that reproduction and publication thereof by Stellenbosch University will not infringe any third-party rights and that I have not previously in its entirety or in part submitted it for obtaining any qualification.

Date: December 2018

Copyright (C) 2018 Stellenbosch University All rights reserved 


\section{TABLE OF CONTENTS}

PART A： LITERATURE REVIEW ..............................................................................1

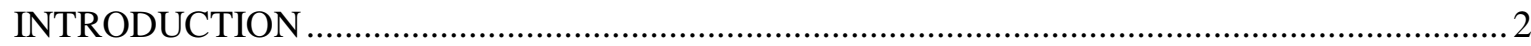

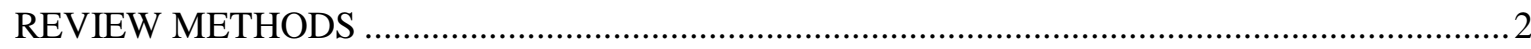

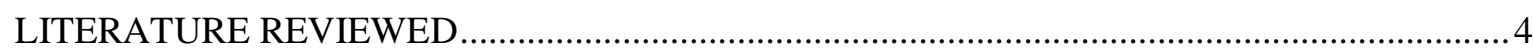

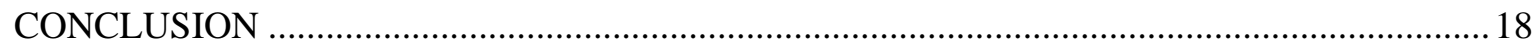

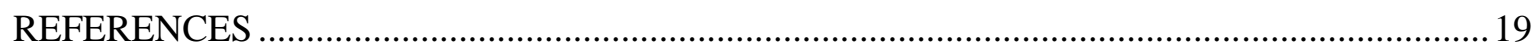

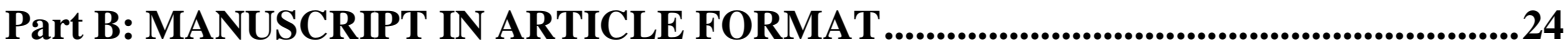

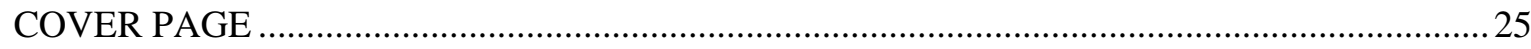

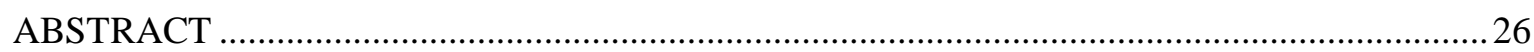

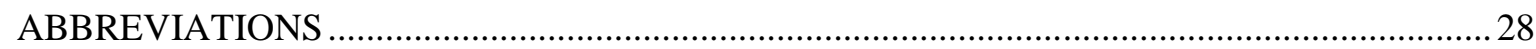

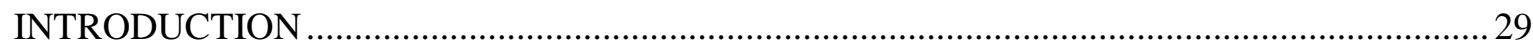

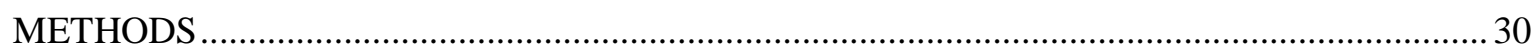

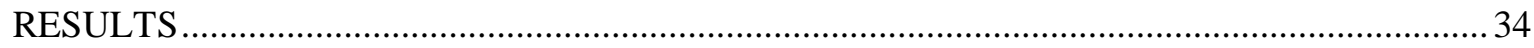

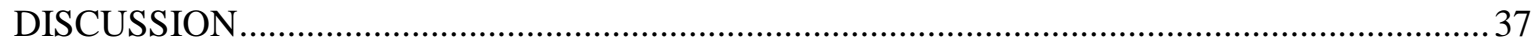

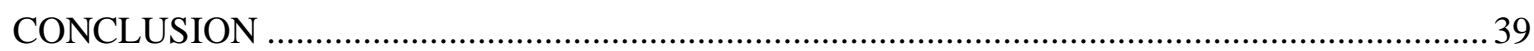

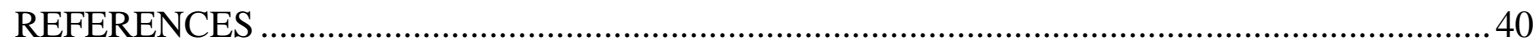

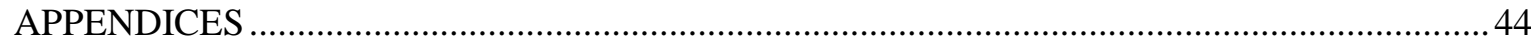

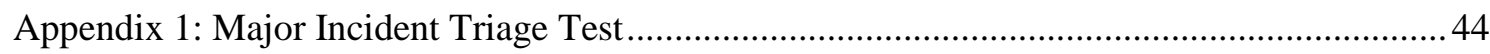

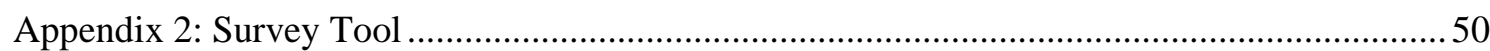

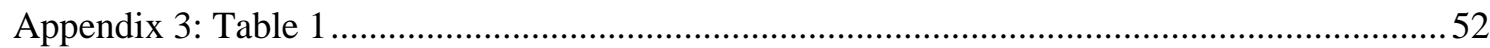

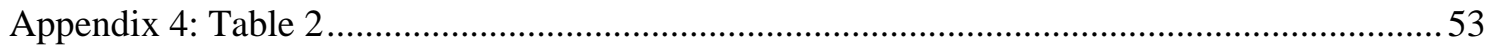

PART C： SUPPORTING DOCUMENTATION ..................................................................54

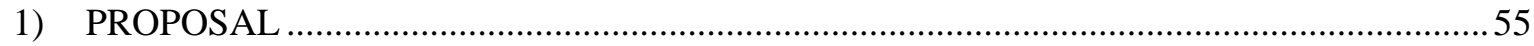

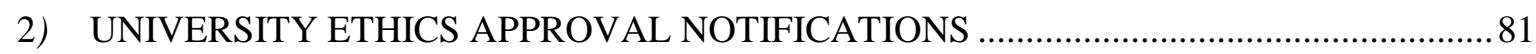

3) APPROVAL FROM WESTERN CAPE DEPARTMENT OF HEALTH ……........................87

4) INSTRUCTIONS FOR CONTRIBUTORS: Prehospital and Disaster Medicine ..................... 88

5) LETTER OF ACCEPTANCE: Prehospital and Disaster Medicine ....................................98 
PART A: LITERATURE REVIEW 


\section{INTRODUCTION}

The Western Cape Emergency Medical Services (EMS) provide 24-hour medical response and pre-hospital medical care routinely and also during major incidents. EMS ambulances are staffed by Basic Life Support (BLS), Intermediate Life Support (ILS) and Advanced Life Support (ALS) prehospital emergency care practitioners. They are the first medical practitioners providing care, including triage, in any major incident. In accordance with the Disaster management objective for integrated institutional capacity, other pre-hospital providers like fire and police receive the same disaster management training as EMS, so that they are able to provide basic care and triage if they arrive first. This integrated institutional approach is also reflected in the command and control structure, where each emergency service is represented in the unified command system. The responsibility of control, falls to a single emergency service; usually the police.

Since South Africa adopted the Major Incident and Medical Support (MIMMS) principles during the 2010 FIFA world cup, there was no study comparing triage accuracy and duration between different levels of prehospital healthcare practitioners using the Triage Sieve algorithm which is used in MIMMS. Triage accuracy is critically important to ensure proper patient management, appropriate referral and resource utilisation

\section{REVIEW METHODS}

\section{Objective}

The objective of this literature review was to assess evidence from existing literature on the current status, practices and recommendations for major incident triage by Prehospital Emergency Medical Teams. In addition, key aspects of the origins of triage, old practices and the evidence behind changes in practice of Major Incident Triage were also included. Comparisons in practice across different countries and health systems were also made. 


\section{Search strategy}

A literature search was undertaken on 1 April 2017 and repeated on 31 January 2018, using the following databases: Science Direct, PubMed central, Google Scholar, Scopus, CINAHL and PLos.

References of identified articles were reviewed and other relevant articles identified and retrieved through Google Scholar Search Engine and the TRIP database.

The following keywords were used: "Prehospital", "Out of Hospital", "Paramedics", “Emergency Medical Technicians", "Prehospital Medical Teams", "Prehospital Emergency Care Practitioners", "Triage", "Triage and Sieve", "Triage and Sort", "Disaster", "Major Incident"," Mass Casualty", “Trauma", "Triage and Performance”, “Triage and Comparison”.

\section{Selection criteria:}

The criteria used to select articles were:

Inclusion criteria:

- Manuscripts describing major incident triage

- Published in peer-reviewed journal in the last 5 years (papers older than 5years were also considered according to relevance to research topic).

Exclusion criteria:

- Published manuscripts in languages other than English.

\section{Quality criteria:}

Titles and abstracts were reviewed to assess relevance to the review objectives and were excluded if they were deemed not applicable. 


\section{LITERATURE REVIEWED}

A major incident is defined as an incident in which the location, number, severity or type of live casualties require extraordinary resources. The impact of the event generates more casualties than locally available resources can manage using routine procedures and require extraordinary means to manage.(1) The ensuing chaotic aftermath typical of major incidents demands organisational and medical prioritisation for effective management. Major incident triage is perhaps one of the single most important activities and serves as a critical management tool that sets the basis for appropriate treatment and referral of patients in order to facilitate appropriately timed life-saving interventions.(2)(3)(4)

The concept of triage arose from the military need to adequately utilize resources when managing the critically injured, in order to get most soldiers back to battle in the shortest possible time. The earliest documentation of triage was around 1792 by the Napoleonic surgeons Dominique Jean Larrey and Francois Percy. Since then, the concept of military triage has seen progressive evolution and refinement for civilian use.(5)(6)

Following Larrey and Percy's concept, a vital addition was made in 1846 to what had then become military triage. Rear Admiral John Crawford Wilson, a British Royal Navy officer, recommended that surgeons should prioritise management of the critically injured that sustained salvageable injuries over the less injured and the fatally injured for whom treatment would not significantly change their outcome.(7) During World War I, another significant development was made in military triage with the introduction of a new key element: resource allocation. Soldiers whose injuries were viable but resource demanding were left to die and resources diverted to managing casualties whose injuries required less time and supplies,(7) essentially doing "the best for the most". It was also during this time that the word "triage" was specifically used for the first time.(8)(9) World War II saw the development and use of a tiered triage system by the United States Army Medical Corps to determine which patients need to be transported 
to higher care centres following initial field treatment. A better structured four tier triage system (minimal, delayed, immediate, and expectant categories) was implemented by the United States Military during the Korean War and with establishment of Mobile Army Surgical Hospitals (MASH) providing resuscitative treatment, 10 miles from the frontlines.

Patients were transported to these centres by helicopter, reducing treatment times to 3 to 12 hours post injury. Subsequently mortality from all wounds decreased to $2.4 \%$. (8) (10) (11)

Triage concepts were eventually adapted for use in civilian medical care in the mid- $19^{\text {th }}$ century. From this time onwards there is increasing documentation of non-military use of triage in hospital emergency departments (12), in routine prehospital settings and in disaster and major incident situations like the 1985 Mexico City Earthquake and the 1987 Amtrak train collision in New York and more recently the 2013 Boston marathon bombing. (13) (2)

The actual practice of triage during major incidents differs from routine prehospital triage and is determined by the type and severity of injuries and the number and distribution of patients.(13)(14) Major incident triage is a continuous and dynamic process with two phases: Primary triage and Secondary triage. Primary triage is carried out at the incident scene to prioritize patients need for treatment and/or evacuation. On the other hand secondary triage is done at the casualty clearing station or hospital emergency department to establish the order in which patients receive care.(10)(14)(15)

\section{Triage performance:}

In major incident management, triage accuracy is critically important and prehospital practitioners are expected to perform quick and accurate triage. Specific triage protocols allow objective assessment and categorisation of patients according to pre-set algorithms in order to improve accuracy and reliability.(16) Triage errors occur when patients are inappropriately classified. Two types of errors can occur during triage: under triage and over triage. Under triage occurs when patients with life threatening injuries requiring immediate treatment are 
inappropriately classified to receive delayed care. On the other hand, over triage occurs when patients with non-critical injuries are classified as urgent and requiring immediate care. This results in inappropriate assignment of resources and can hinder the effective management of critically injured patients.(9)(17)

Understanding the reasons for triage errors is key to improving the triage process and accuracy. Just like the chaotic aftermath of a major incident, reasons for errors are numerous and varied and range from age of victims (18), lack of memory aides, documentation and tags (19) to choice of algorithm and the level of skill of the user. (20) However, choice of algorithm and skill level of user are the main reasons and have been proven in a real life major incident. During the 2005 London bombings, the main factors responsible for the effective response and low critical mortality rate $(15 \%)$ was that a simplified triage algorithm used by an appropriately skilled prehospital medical team.(21)

\section{Triage algorithms}

Triage, like many other clinical decision challenges can be mitigated using algorithms. An algorithm is a clinical guideline that provides a simplified standardized step by step decision making tool to avoid errors. (22) Evidence shows that guideline-driven care is effective in changing the process and outcome of care provided by professions allied to medicine Major incident triage performance relies not only on the type of algorithm but also the knowledge and skills level of the person carrying out the triage. (23)

\section{Different triage algorithms}

There are currently various non-military major incident triage systems used around the world for both primary and secondary triage. The most commonly used primary triage algorithms include START (Simple Triage and Rapid Treatment), Sacco Triage Method (STM), Care Flight triage and Triage Sieve.(24) Newer algorithms like the Primary Ranking for Initial 
Orientation in Emergency Medical Services (PRIOR) and Amberg-Schwandorf Algorithm for Triage (ASAV) have also been introduced.(24) However, there is no convincing scientific evidence to support the use of any one of the existing systems over another. The choice of major incident triage algorithm remains largely locally or institutionally determined. (10)(14)

The START triage system (Figure 1) was developed in 1983 and utilises objective physiological data to allocate patients to one of four categories which dictate their treatment priority. The categories are 1) deceased or expectant, 2) immediate, 3) delayed, and 4) minor (ambulatory). (10)

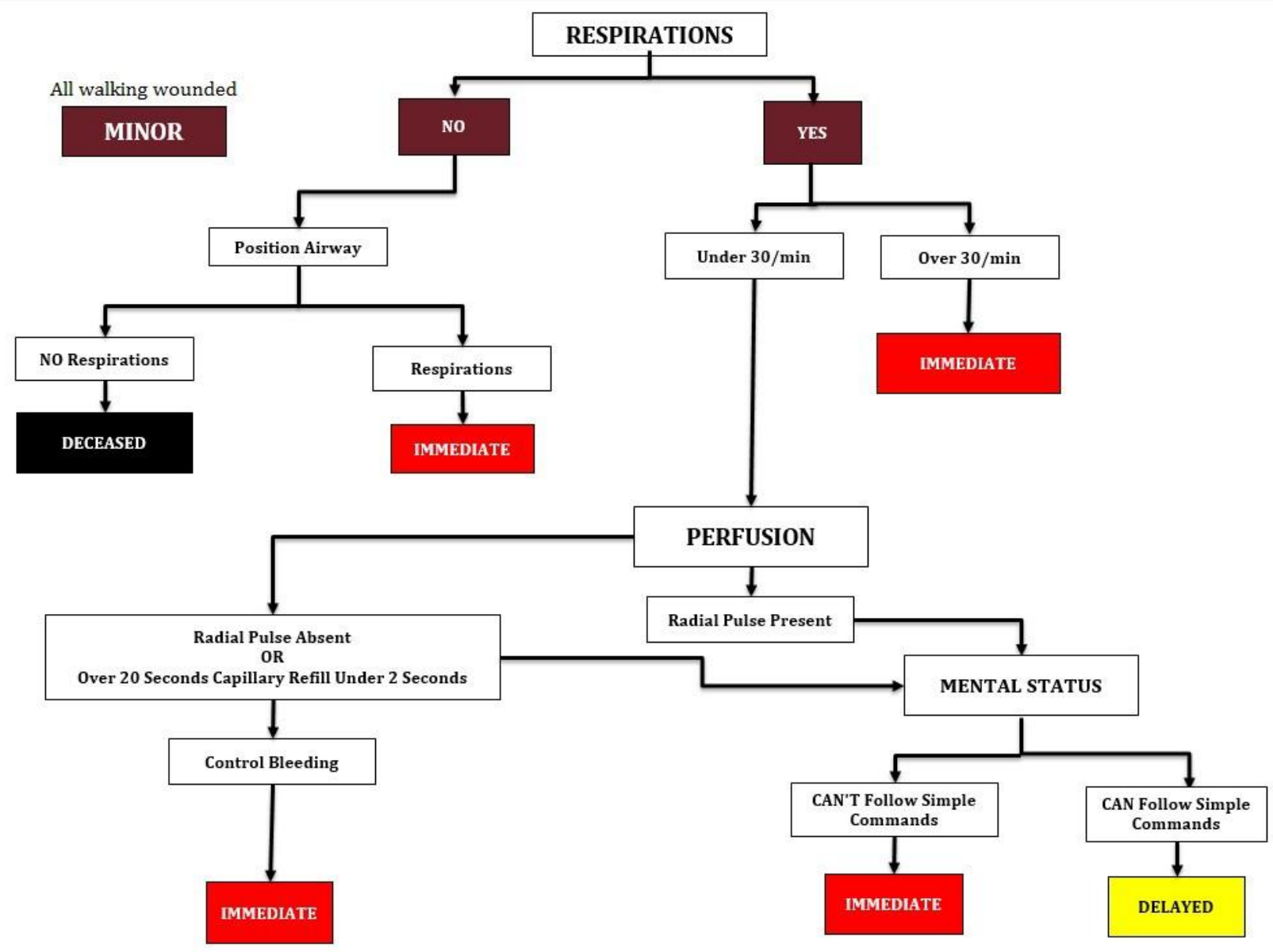

The START (Simple Treatment and Rapid Transport) Triage Algorithm

Figure 1: The START (Simple Treatment and Rapid Transport) triage algorithm 
The START algorithm is largely used by Emergency Medical Services (EMS) in the United States and has been implemented during major incidents such as the 1989 Northridge earthquake, the 1995 Oklahoma City bombing, and the 2001 attacks on the New York World Trade Centre.(10) However, limitations of the START algorithm include the lack of an outcome measure; no consideration of resource availability coupled with poor utilisation of resources; lack of inter-category victim prioritisation; lack of consideration of victim deterioration, prognosis or different trauma types; and infrequent use impacting triage accuracy. (26)

Modified START (Figure 2) is an algorithm derived from START triage, whereby a new "Orange" category is added between the immediate (red) category and the delayed (yellow) category. In a study comparing modified START to START algorithms, the former performed better with correct triage of $86.3 \%$ of cases (over-triage $1.5 \%$; under-triage $12.2 \%$ ), compared to $81.5 \%$ of cases (over-triage $17.3 \%$; under-triage $1.3 \%$ ), a difference of $4.9 \%$ (95\% CI, 1.5 8.2). While the difference in overall triage performance was not significant, the over-triage rate of START (11.5 times higher than modified START) would make it less suitable to low resource settings as resources would be utilised by non-critical patients 


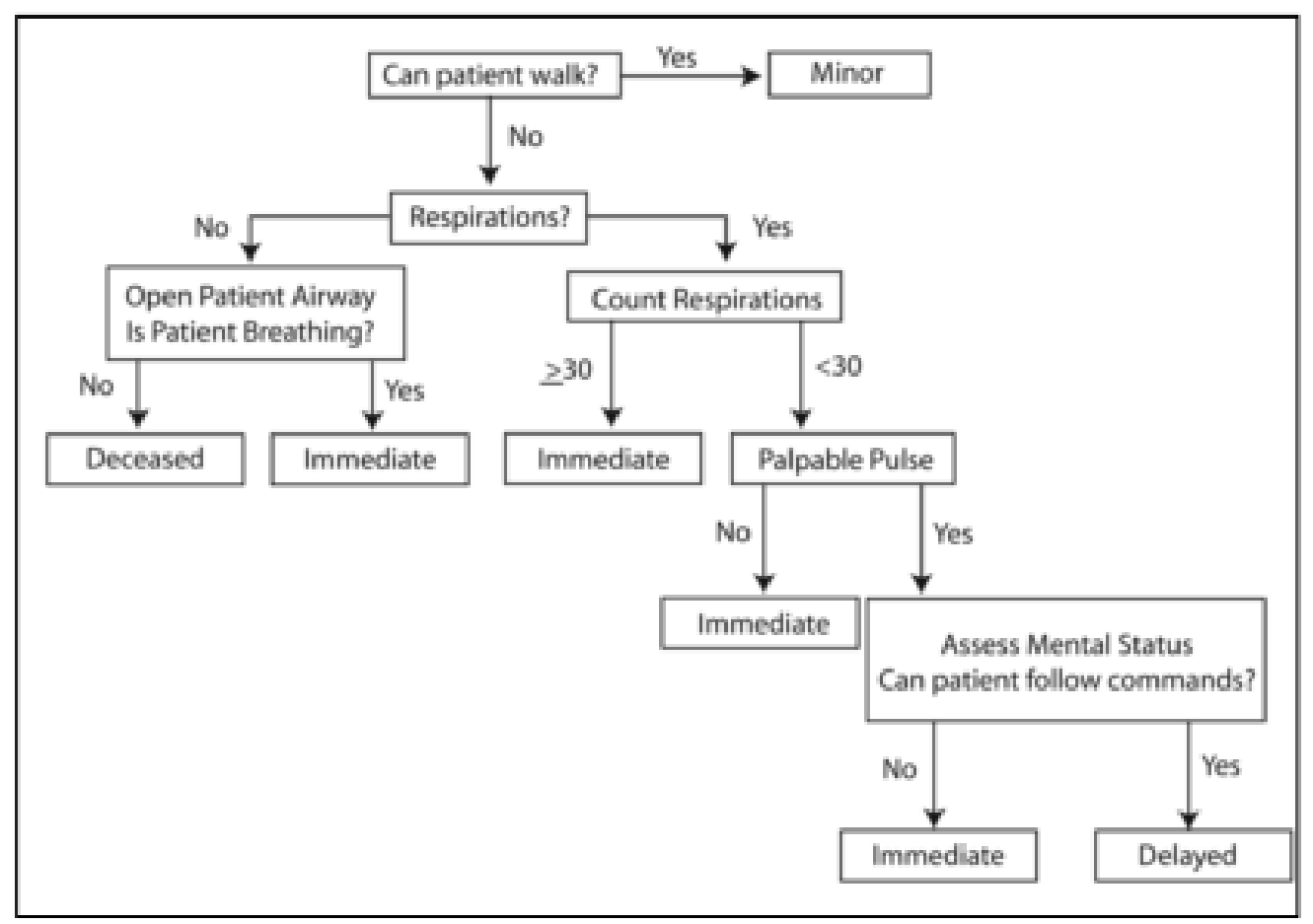

Figure 2: The modified START triage algorithm (10)

Romig et al. developed a paediatric version of START in 2002 called the jumpSTART algorithm (Figure 3). It is for use in major incidents that involve children (1-8 years) and it takes into consideration the unique attributes of paediatric physiology. The jumpSTART algorithm uses the same colour coding as the START, however it allows for first responders to give 5 rescue breaths in apnoeic children with palpable pulses. There is no literature available describing the use of the jumpSTART algorithm in a real disaster situation. (10)(28) 


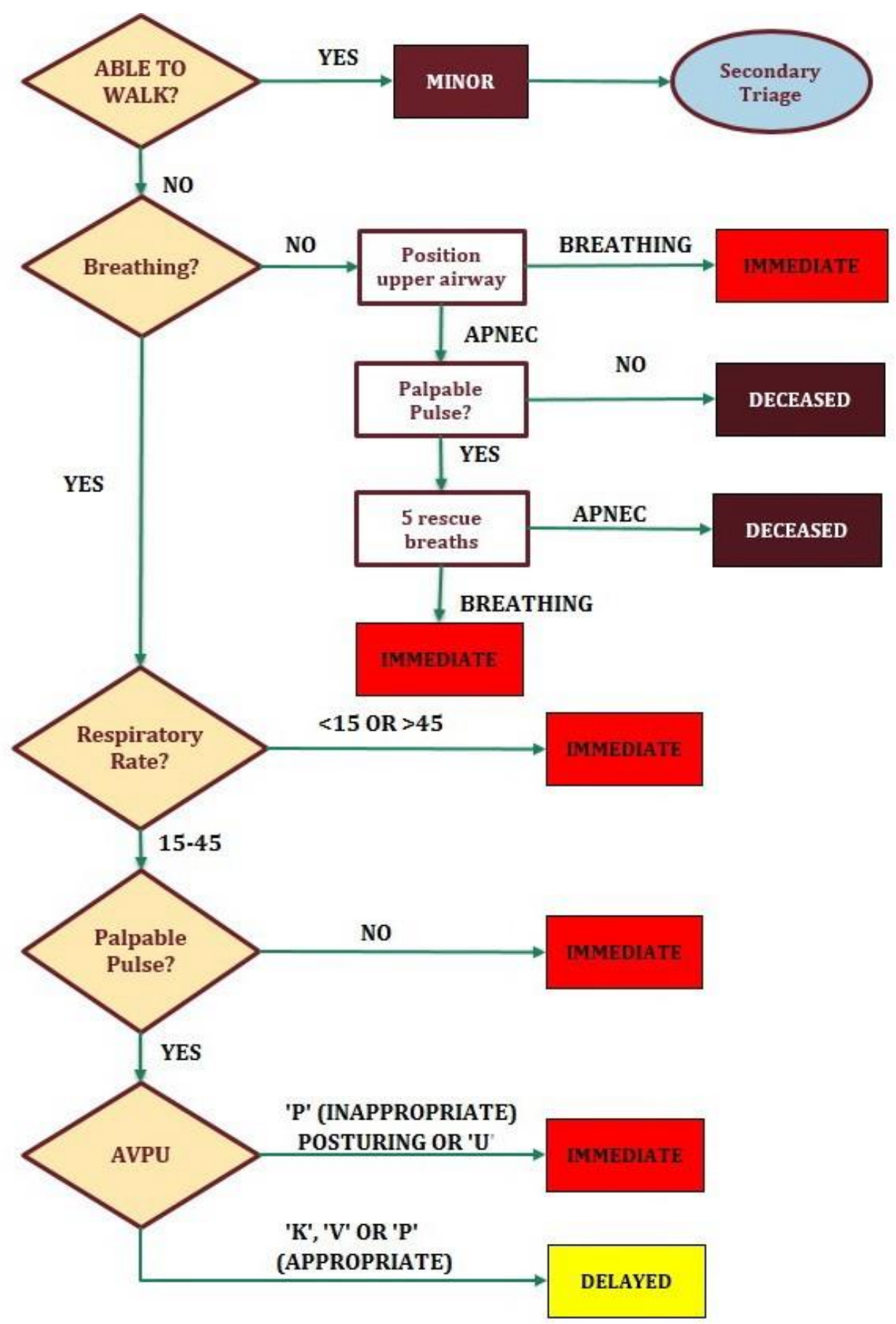

Figure 3: The jumpSTART triage algorithm

The Triage Sieve system was introduced in 1995 by Hodgetts and Mackway-Jones as part of the Major Incident Medical Management and Support (MIMMS) course for healthcare providers.(30) It is preferred by providers in the United Kingdom, parts of Australia(31) and 
South Africa. (15) The Triage Sieve algorithm assigns patients to one of three priority categories using various physiological parameters (Figure 4). (3) The categories are: Priority 1

/ Immediate - Require immediate life-saving intervention; Priority 2 / Urgent - Require medical or surgical intervention within 2-4 hours; and Priority 3 / Delayed - Treatment can be delayed $>4$ hours.

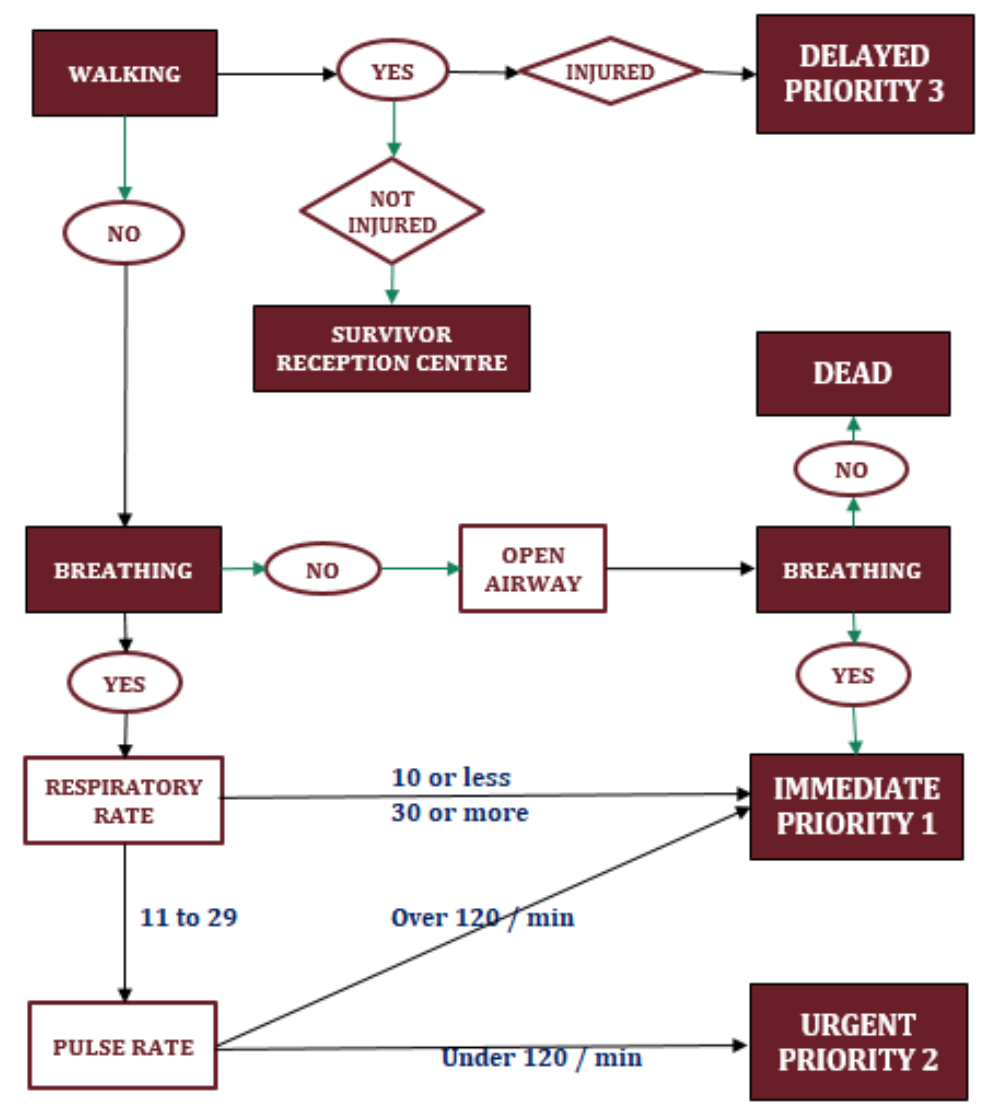

\section{Figure 4: The Triage Sieve algorithm}

Unlike the START triage system, Triage Sieve does not formally assess the mental status of victims. In addition, Triage Sieve defines a respiratory rate of less than 10 breaths/minute or more than 30 breaths/minute as abnormal, while START only considers more than 30 breaths/minute as abnormal. The START algorithm also only assesses for the presence or absence of a pulse while the Triage Sieve algorithm includes the rate, with more than 120 
beats/minute or a Capillary Refill Test (CRT) of more than 2 seconds as being abnormal.(10)(15) Triage Sieve, like other algorithms that use ability to walk as an initial assessment, allow rapid evaluation and their simplicity allows for use by non-medical personnel. Nonetheless, they are prone to over triage immobile patients and under triage severely injured patients who can walk.(32)(33)

The Triage Sieve algorithm was modified by Hodgets and his colleagues for use in major incidents involving children. The Paediatric Triage Tape (PTT) is a waterproof tape that uses the child's length to provide age-adjusted parameters of the Triage Sieve.(34)

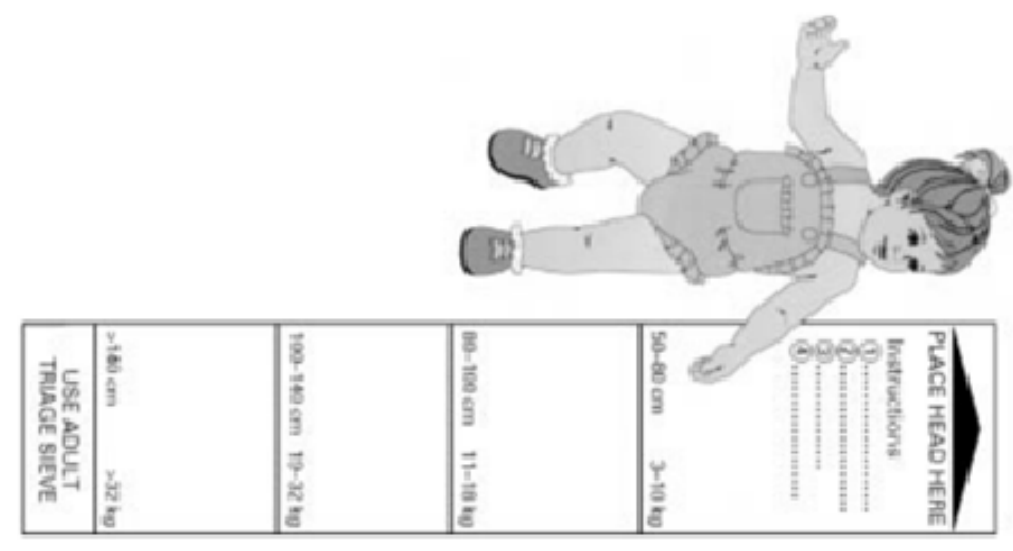

\section{Figure 5: Using the Triage Tape (33)}

Nocera and Garner developed the CareFlight Triage algorithm in 2001 (Figure 6). The algorithm was intended to standardise major incident triage by Australian first responders. (10)(35) 


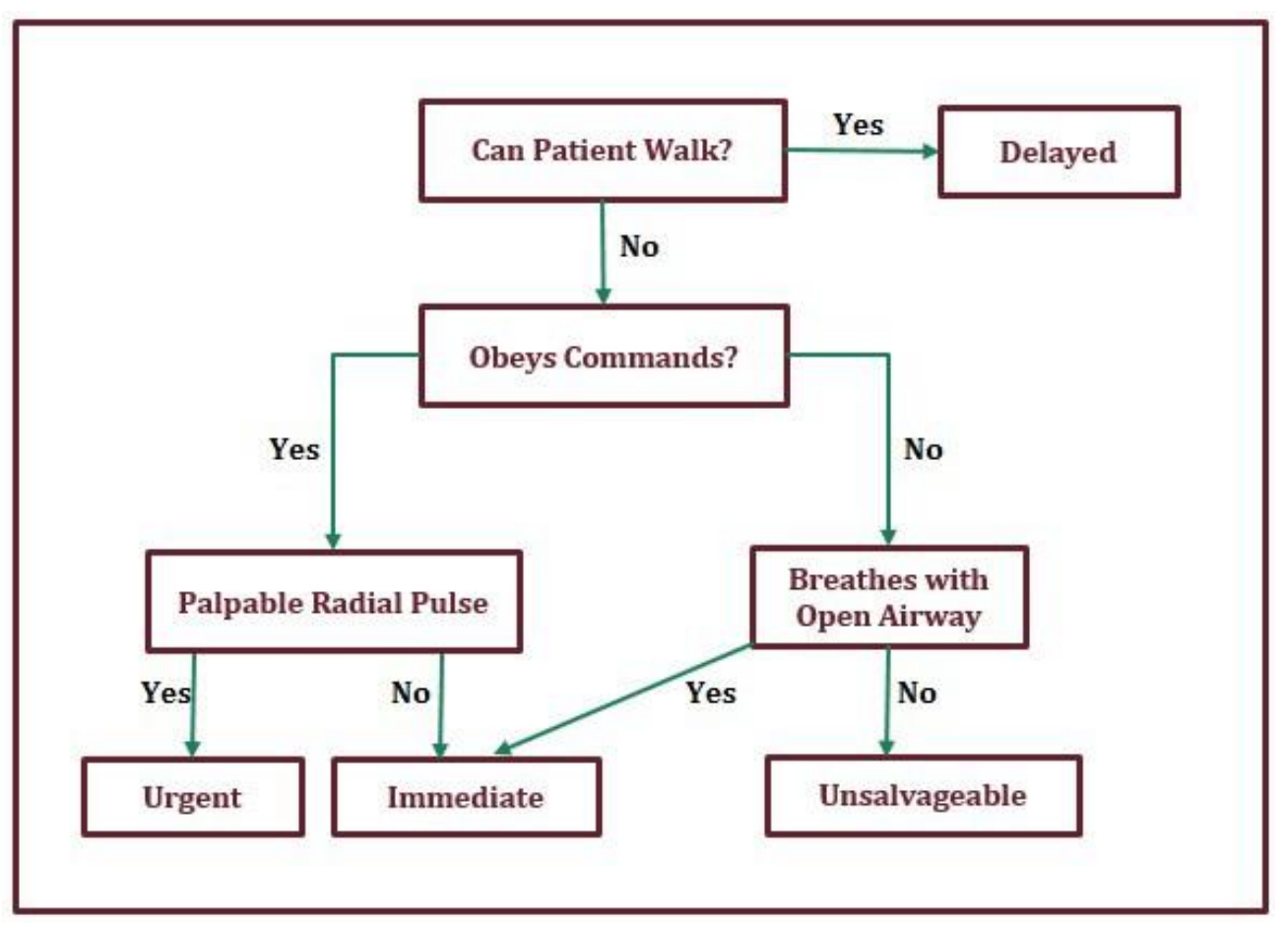

Figure 6: The CareFlight Triage Algorithm

The CareFlight algorithm, similar to the START system, also sort patients into priority categories by assessing mental status, breathing and palpable radial pulse. However, mental status is assessed first and the actual rate of respiration is not considered. (10) In a retrospective analysis, the ability to predict critical injuries in designated trauma patients was compared between CareFlight, START and modified START algorithms. The results below show that while the CareFlight Triage algorithm had a similar sensitivity to START and modified START algorithms, it had a better specificity overall. (35)

Table 1: Comparison of different triage tools to predict critical injuries

\begin{tabular}{|l|l|l|}
\hline & Sensitivity & Specificity \\
\hline CareFlight & $\begin{array}{l}82 \%(95 \% \text { confidence interval } \\
[\mathrm{CI}]) 75 \% \text { to } 88 \%)\end{array}$ & $96 \%(95 \%$ CI $94 \%$ to $97 \%)$ \\
\hline START & $85 \%(95 \%$ CI $78 \%$ to $90 \%)$ & $86 \%(95 \%$ CI $84 \%$ to $88 \%)$ \\
\hline Modified START & $84 \%(95 \%$ CI $76 \%$ to $89 \%)$ & $91 \%(95 \%$ CI $89 \%$ to $93 \%)$ \\
\hline
\end{tabular}


A major advantage of the CareFlight algorithm is its appropriateness in all age groups. (10) The CareFlight method outperformed the Paediatric Triage Tape, the START algorithm, and the JumpSTART algorithm in a prospective comparison of paediatric major incident primary triage tools (Table 1). (36)

Table 2: $\quad$ Comparison of Paediatric major incident primary triage tools (36)

Table 2 Comparative analysis of MI primary triage algorithms: results by ISS, NISS, and presence of one or more modified Garner criteria

\begin{tabular}{|c|c|c|c|c|c|c|}
\hline & \multicolumn{2}{|c|}{ ISS $>15$} & \multicolumn{2}{|c|}{ NISS $>15$} & \multicolumn{2}{|c|}{ Garner } \\
\hline & $\%$ & $95 \% \mathrm{Cl}$ & $\%$ & $95 \% \mathrm{Cl}$ & $\%$ & $95 \% \mathrm{Cl}$ \\
\hline \multicolumn{7}{|l|}{ PTT } \\
\hline Sensitivity & 37.8 & 32.7 to 42.5 & 26.1 & 23 to 28.8 & 41.5 & 36.8 to 45.6 \\
\hline Specificity & 98.6 & 98.3 to 98.8 & 98.9 & 98.5 to 99.1 & 98.9 & 98.6 to 99.2 \\
\hline \multicolumn{7}{|l|}{ CareFlight } \\
\hline Sensitivity & 48.4 & 43.4 to 52.8 & 31.5 & 28.5 to 34.1 & 46 & 41.2 to 50.2 \\
\hline Specificity & 98.8 & 98.6 to 99.1 & 99 & 98.7 to 99.3 & 98.9 & 98.6 to 99.1 \\
\hline \multicolumn{7}{|l|}{ JumpSTART* } \\
\hline Sensitivity & 3.2 & 1.3 to 7.5 & 2.4 & 1 to 5 & 0.8 & 0.1 to 4.1 \\
\hline Specificity & 97.8 & 97.7 to 98 & 97.8 & 97.6 to 98 & 97.7 & 97.6 to 97.8 \\
\hline \multicolumn{7}{|l|}{ START† } \\
\hline Sensitivity & 31.3 & 21.5 to 42.8 & 22.3 & 15.6 to 30.7 & 39.2 & 29.3 to 50 \\
\hline Specificity & 77.9 & 77.3 to 78.7 & 77.3 & 76.6 to 78.3 & 78.7 & 77.9 to 79.5 \\
\hline
\end{tabular}

The Sacco Triage Method (STM) was developed in 2005 to mitigate the limitations of START and methods similar to it. STM is not an algorithm but rather an empirically derived method that uses mathematical and operational research concepts and combines them into a linear programming model that can be used to determine the order in which victims are transported and treated.(10)(26) It then uses this information to predict the possible number of survivors given the available resources. However, the STM requires specific software and technology support plus constant updates on available resources. This limits its practicality and utilisation in low resource settings. (26) The evidence related to STM is from simulations, and there are no reports on its actual use in a major incident. 
In 2006, the National Association of EMS Physicians (NAEMSP) and the Centres for Disease Control and Prevention (CDC) funded the Sort Assess Life saving interventions Transport

(SALT) workgroup to examine existing literature related to mass casualty triage and make recommendations for a national standard for mass casualty triage in the United States. They developed an all-hazards algorithm applicable to all age groups that could be adapted to different settings. Unlike START and similar algorithms, the SALT algorithm categorises patients into five different groups according to their treatment or transport needs: 1) immediate

2) expectant 3) delayed 4) minimal 5) dead. In addition, SALT includes lifesaving interventions as part of the algorithm (Figure 7).

A

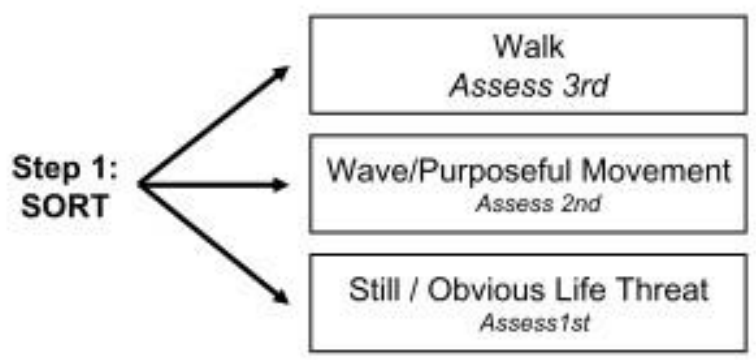

B

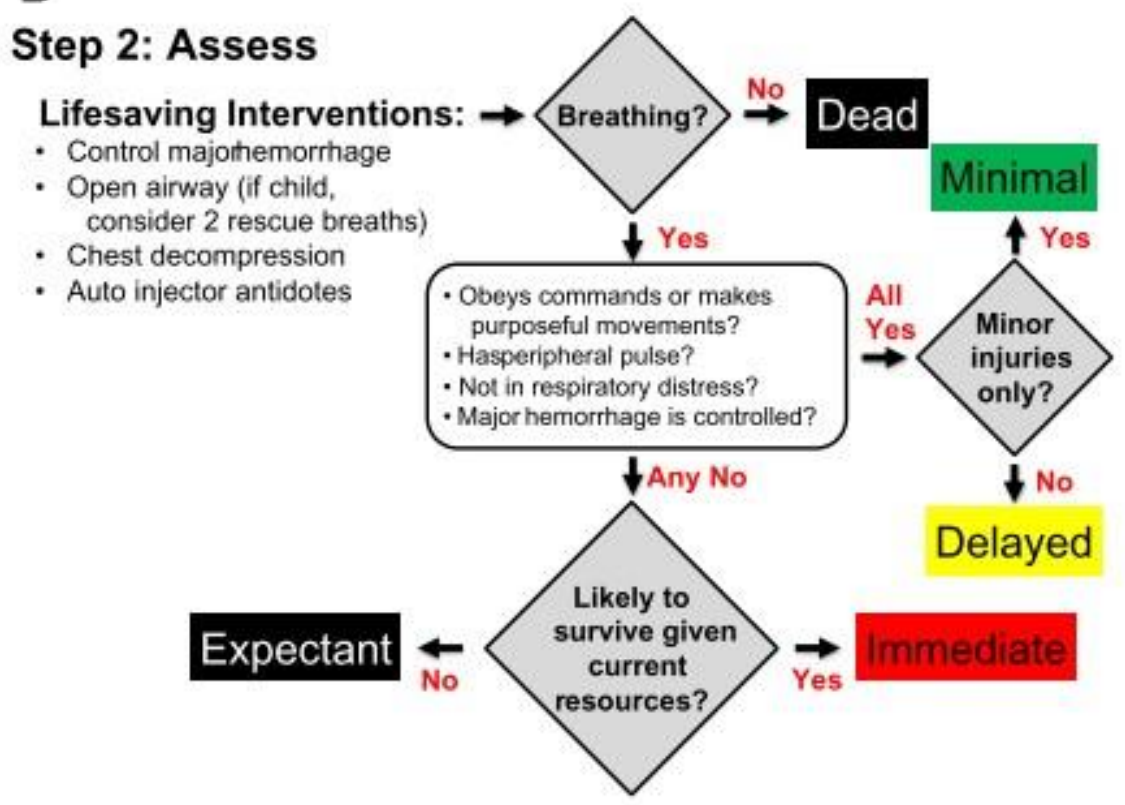

Figure 7: The SALT (Sort Assess Life Saving) triage algorithm (25) 
The system faced a lot of resistance as it required all local, state and federal agencies to change their existing systems. The SALT workgroup then recommended the formation of the Model Uniform Core Criteria (MUCC); consisting of 24 essential elements to include in an ideal Mass Casualty Incident (MCI) triage system. The MUCC allow for interoperability between the different existing triage algorithms. (14)(36)(37)

Primary Ranking for Initial Orientation in Emergency Medical Services (PRIOR) and the Amberg-Schwandorf Algorithm for Triage (ASAV) are recently developed major incident triage algorithms that are primarily used in Germany. Under the guidance of the German Society for Disaster Medicine, PRIOR was developed for use in both injured and non-injured patients. In a recent German study, the diagnostic precision of PRIOR was compared to other primary triage algorithms.(24) Although the PRIOR algorithm had the highest sensitivity $(90 \%)$, its low specificity of only $54 \%$ resulted in it being outperformed by other triage algorithms (overall START performed the best). The deliberate inclusion of both injured and non-injured patients is unique, but it is time consuming. (24)

The Amberg-Schwandorf Algorithm for Triage (ASAV) was designed for use by nonphysician EMS personnel in physician based EMS systems. It is used in Germany for primary triage by non-physicians followed by a mandatory secondary triage on scene by a prehospital emergency physician. In a German simulation study, ASAV reliably detected red patients (requiring immediate treatment and/or transport) with a sensitivity of $87 \%$ and specificity of $91 \%$. (22) However, it was still outperformed by other major incident triage tools in a direct comparative study. (24)

\section{Evidence for triage algorithms}

A significant proportion of the science and evidence in major incident triage is generated from methodologies on the lower tiers of the pyramid of scientific evidence. These include simulated 
scenarios, paper based exercises, expert-opinions and case reports. Therefore, recommendations and conclusions drawn from these studies are of limited value. Majority of the current triage algorithms are tailored towards physiological derangements in injured victims. Further research is needed to address use of triage algorithms in non-injury major incidents like CBRN (Chemical Biological Radiological Nuclear) and the incorporation of existing resources in Major incident triage decision making. Given that major incidents occur and are managed locally, health systems should invest in studies to identify which algorithms are best suited to their environment. In South Africa, a national major incident triage system was introduced in 2010 as part of the Fédération Internationale de Football Association (FIFA) world cup preparations. The South African National Department of Health adopted the Major Incident Medical Management and Triage Support System (MIMMS), which originated in the UK. The system utilised in MIMMS is that of sieve and sort. Triage Sieve occurs at the site of the incident to prioritize patients in the field for evacuation and transport to definitive medical care while sort is at patient referral sites and establishes the order in which patients receive care. (15)

\section{Influence of skill level on triage accuracy}

A significant part of improving triage accuracy, is the choice of triage officer. Numerous research studies have been done to evaluate which skill's level is best suited to perform triage.

In one study, Lee et al. compared triage accuracy amongst trainees of three different categories of first responders: Prehospital medical personnel, Fire personnel and Police personnel. Participants triaged written major incident scenarios after receiving training using the SALT triage system. Initial test scores were higher for the Prehospital medical personnel (87.0\%) compared to fire personnel $(80.2 \%)$ and police personnel $(68.0 \%)$. The test was repeated after three months and a similar trend was noticed (prehospital medical personnel 75.4\%; fire 71.4\%, police $57.8 \%$ ). Another study indicated that first-year medical students attained accuracy scores similar to qualified emergency physicians, registered nurses and paramedics after a brief training session. (38) 
Fitzharris et al conducted a retrospective evaluation of adherence to the New South Wales (NSW) prehospital triage protocol amongst the different skill levels of prehospital medical personnel in the ambulance service. This study evaluated protocol adherence as one of the reasons for errors in the triage of patients. The highest protocol adherence rates (77\%) were found among the lowest-level trained personnel.(39)

In South Africa, routine prehospital triage is done by prehospital personnel of three different skill levels: Basic Life Support (BLS), Intermediate Life Support (ILS) and Advanced life Support (ALS). The South African Triage Score (SATS) (40) is used to prioritise patients in routine situations where both time and resources are adequate to attend to individual patients. (1) However, during major incidents the additional stress and chaotic environment makes triage more difficult. Furthermore, a different algorithm from the routine one is used. The personnel who routinely triage, should triage in a major incident as their experience and familiarity should make them more efficient.(1)(41) However, as with the New South Wales study (29), the triage performance between differently skilled prehospital personnel differs and it is thus important to identify the most appropriate personnel to ensure the most accurate triage performance.

\section{CONCLUSION}

Major incident triage has undergone extensive developments and changes over the centuries as societies become more complex and major incidents increase. Numerous triage algorithms have been developed as tools to improve the process of quickly identifying individuals who would most likely benefit from rapid medical attention. However, many factors influence effective utilisation of these tools, key amongst them being the skills level of the triage officer. 


\section{REFERENCES}

1. Baker MS. Creating order from chaos: part I: triage, initial care, and tactical considerations in mass casualty and disaster response. Mil Med. 2007; 172(3):232-6.

2. Gates JD, Arabian S, Biddinger P, et al. The Initial Response to the Boston Marathon Bombing: Lessons Learned to Prepare for the Next Disaster. Annals of surgery. 2014; 260(6):960-966.

3. Vassallo J, Smith JE, Bruijns SR, Wallis LA. Major incident triage: A consensus based definition of the essential life-saving interventions during the definitive care phase of a major incident. Injury. 2016;47(9):1898-902.

4. Ingrassia PL, Prato F, Geddo A, Colombo D, Tengattini M, Calligaro S, et al. Evaluation of Medical Management During a Mass Casualty Incident Exercise: An Objective Assessment Tool to Enhance Direct Observation. J Emerg Med. 2010;39(5):629-36.

5. $\quad$ Edwards M. Triage. Lancet. 2009;373(9674):1515.

6. Falzone E, Pasquier P, Hoffmann C, Barbier O, Boutonnet M, Salvadori A, et al. Triage in military setting. Anaesth Crit Care Pain Med. 36(1):43-51.

7. Iserson K V., Moskop JC. Triage in Medicine, Part I: Concept, History, and Types. Ann Emerg Med. 2007; 49(3):275-81.

8. Sharma BR. Triage in trauma-care system: A forensic view. J Clin Forensic Med. 2005;12(2):64-73.

9. Frykberg ER. Triage: Principles and practice. Scand J Surg. 2005;94(4):272-8.

10. Jenkins JL, McCarthy ML, Sauer LM, Green GB, Stuart S, Thomas TL, et al. Masscasualty triage: time for an evidence-based approach. Prehosp Disaster Med. 2008;23(1):3-8.

11. Driscoll RS. New York Chapter History of Military Medicine Award. U.S. Army medical helicopters in the Korean War. Mil Med. England; 2001 Apr;166(4):290-6. 
12. Welch SJ, Davidson SJ. The Performance Limits of Traditional Triage. Ann Emerg Med. 2011;58(2):143-4.

13. Leaning J. PHYSICIANS, TRIAGE, AND NUCLEAR WAR. Lancet. 1988;332(8605):269-70.

14. Klein KR, King R V, Lehman T. Qualitative Analysis of Surveyed Emergency Responders and the Identified Factors That Affect First Stage of Primary Triage Decision Making of Mass Casualty Incidents. PLoS Curr. (19):8.

15. Smith W. Triage in mass casualty situations. Cme. 2012;30(11):414.

16. Martí R, Robles S, Martín-Campillo a., Cucurull J. Providing early resource allocation during emergencies: The mobile triage tag. J Netw Comput Appl. 2009;32(6):1167-82.

17. Frykberg ER. Medical management of disasters and mass casualties from terrorist bombings: how can we cope? J Trauma. United States; 2002 Aug;53(2):201-12.

18. Rehn M, Eken T, Kruger AJ, Steen PA, Skaga NO, Lossius HM. Precision of field triage in patients brought to a trauma centre after introducing trauma team activation guidelines. Scand J Trauma Resusc Emerg Med. 2009;17(1).

19. Rådestad M, Lennquist Montán K, Rüter A, Castrén M, Svensson L, Gryth D, et al. Attitudes Towards and Experience of the Use of Triage Tags in Major Incidents: A Mixed Method Study. Prehosp Disaster Med. Cambridge University Press; 2016 Aug 23;31(4):37685.

20. Veen M van, Steyerberg Ewout W, Ruige Madelon, Meurs Alfred H J van, Roukema Jolt LJ van der et al. manchester triage system in paediatric emergency care prospective observational study. BMJ. :337:a1501. 
21. Aylwin CJ, König TC, Brennan NW, Shirley PJ, Davies G, Walsh MS, et al. Reduction in critical mortality in urban mass casualty incidents: analysis of triage, surge, and resource use after the London bombings on July 7, 2005. Lancet. 2006;368(9554):2219-25.

22. Wolf P, Bigalke M, Graf BM, Birkholz T, Dittmar MS. Evaluation of a novel algorithm for primary mass casualty triage by paramedics in a physician manned EMS system: A dummy based trial. Scand J Trauma Resusc Emerg Med. 2014;22(1):1-10.

23. Carron P, Taffe P, Ribordy V, Schoettker P, Fishman D, Yersin B. Accuracy of prehospital triage of trauma patients by emergency physicians : a retrospective study in western Switzerland. Eur J Emerg Med. 2011;18(2):86-93.

24. Heller AR, Salvador N, Frank M, Schiffner J, Kipke R, Kleber C. Diagnostic quality of triage algorithms for mass casualty incidents. Anaesthesist. Germany; 2017 Oct;66(10):762-72.

25. Bhalla MC, Frey J, Rider C, Nord M, Hegerhorst M. Simple Triage Algorithm and Rapid Treatment and Sort, Assess, Lifesaving, Interventions, Treatment, and Transportation mass casualty triage methods for sensitivity, specificity, and predictive values. The American Journal of Emergency Medicine. 2015.

26. Sacco WJ, Navin DM, Fiedler KE, Waddell RK 2nd, Long WB, Buckman RFJ. Precise formulation and evidence-based application of resource-constrained triage. Acad Emerg Med. United States; 2005 Aug;12(8):759-70.

27. Arshad FH, Williams A, Asaeda G, Isaacs D, Kaufman B, Ben-Eli D, et al. A Modified Simple Triage and Rapid Treatment Algorithm from the New York City (USA) Fire Department. Prehosp Disaster Med. 2015;30(2):199-204.

28. LE R. Pediatric triage. A system to JumpSTART your triage of young patients at MCIs. JEMS. 2002;27(7):52-3. 
29. Waisman Y, Amir L, Mor M, Feigenberg Z, Aharonson LD, Peleg K, et al.

Prehospital Response and Field Triage in Pediatric Mass Casualty Incidents: The Israeli Experience. Clin Pediatr Emerg Med. 2006;7(1):52-8.

30. Mackway-Jones K. Major Incident Medical Management and Support: The Practical Approach at the Scene. In Wiley-Blackwell; 2011. p. 89-102.

31. Nocera A, Garner A. Australian disaster triage: A colour maze in the Tower of Babel. Aust N Z J Surg. 1999;69(8):598-602.

32. Castle N. Triage and transport decisions after mass casualty incidents. Emerg Nurse. 14(1):22-5.

33. Lax P, Prior K. Major incident pre-hospital care. Surg. Elsevier Ltd; 2015; Volume 33(Issue 9):419-23.

34. Hodgetts TJ, Hall J, Maconochie I SC. Pediatric Triage Tape. Prehospital Immed Care. 1998;2:155-159.

35. Garner A, Lee A, Harrison K, Schultz CH. Comparative analysis of multiple-casualty incident triage algorithms. Ann Emerg Med. American College of Surgeons, Chicago, IL; 2001;38(5):541-8.

36. Wallis LA, Carley S. Comparison of paediatric major incident primary triage tools. Emerg Med J. England; 2006 Jun;23(6):475-8.

37. Lerner EB, Mckee CH, Cady CE, Cone DC, Colella MR, Cooper A, et al. A Consensus-Based Gold Standard for the Evaluation of Mass Casualty Triage Systems. Prehospital Emerg Care. 2015;19(2):267-71. 
38. Sapp RF1, Brice JH, Myers JB, Hinchey P.Sapp RF1, Brice JH, Myers JB HP. Triage performance of first-year medical students using a multiple-casualty scenario, paper exercise. Prehosp Disaster Med. 25(3):239-45.

39. Fitzharris M, Stevenson M, Middleton P, Sinclair G. Adherence with the pre-hospital triage protocol in the transport of injured patients in an urban setting. Injury. Elsevier Ltd; 2012;43(9):1368-76.

40. Twomey M, Wallis L a., Thompson M Lou, Myers JE. The South African triage scale (adult version) provides valid acuity ratings when used by doctors and enrolled nursing assistants. African J Emerg Med. 2012;2(1):3-12.

41. Aitken P, Fitzgerald G, Hospital TT. Disaster triage : Evidence, consistency and standard practice. $2012 ; 24(3): 222-4$. 


\section{Part B: MANUSCRIPT IN ARTICLE FORMAT}

As accepted by Prehospital Disaster Medicine journal 


\section{A comparison between differently skilled pre-hospital emergency care providers in major incident triage in South Africa}

Author information:

1. Annet Alenyo Ngabirano

MBChB (MUST), MSc Dis.Med (EMDM)

Division of Emergency Medicine, Stellenbosch University

(Corresponding author: 16 Benona Rd, Lansdowne, Cape Town, aalenyo@yahoo.com)

2. Wayne P. Smith

BSc, MBChB, MSc Dis.Med (EMDM), FCEM (SA)

Division of Emergency Medicine, University of Cape Town

3. Michael McCaul

Biostatistics Unit, Centre for Evidence-based Health Care

Division of Epidemiology and Biostatistics, Stellenbosch University

4. Daniel J. Van Hoving

MBChB, DipPEC (SA), MMed (EmMed), MScMedSci (ClinEpi)

Division of Emergency Medicine, Stellenbosch University

Word count: 2,920

Number of figures: 3

Number of tables: 2 


\begin{abstract}
Introduction

Major incident triage ensures effective emergency care and utilization of resources. Prehospital emergency care providers are often the first medical professionals to arrive at any major incident and should be competent in primary triage. However, various factors including level of training influence their triage performance.
\end{abstract}

\title{
Hypothesis/Problem
}

To determine the difference in major incident triage performance between different training levels of prehospital emergency care providers in South Africa utilizing the Triage Sieve algorithm.

\section{Methods}

A cross sectional study involving differently trained prehospital providers; advanced life support (ALS), intermediate life support (ILS), and basic life support (BLS). Participants wrote a validated 20 question pre-test before completing major incident training. Two post-tests were also completed; a 20 question written test and a 3 question face-to-face evaluation. Outcomes measured were triage accuracy and duration of triage. The effect of level of training, gender, age, previous major incident training and duration of service were determined.

\section{Results}

A total of 129 prehospital providers participated. The mean age was 33.4 years and $65(50.39 \%)$ were male. Most ( $\mathrm{n}=87,67.44 \%)$ were BLS providers. The overall correct triage score pretraining was $53.91 \%$ (95\% CI 51.98 to 55.83 ), over triage $31.43 \%$ (29.66 to 33.2 ) and under triage $13.84 \%$ (12.55 to 12.22$)$. Post-training, the overall correct triage score increased to $63.60 \%$ (61.72 to 65.44$)$, over triage decreased to $17.91 \%$ (16.47 to 19.43$)$ and under triage 
increased to $17.83 \%$ (16.40 to 19.36). ALS providers had both the highest likelihood of a correct triage score post-training (odds ratio 1.21 (95\%CI 0.96-1.53)) and the shortest duration of triage (median $3 \mathrm{sec}$, interquartile range 2 to $7 \mathrm{sec})(\mathrm{p}=0.034)$. Participants with prior major incident training performed better $(\mathrm{p}=0.001)$.

\section{Conclusion}

Accuracy of major incident triage across all levels of prehospital providers in South Africa is less than optimal with non-significant differences post major incident training. Prior major incident training played a significant role in triage accuracy indicating that training should be an ongoing process. Although ALS-providers were the quickest to complete triage, this difference was not clinically significant. BLS and ILS providers with major incident training can be thus be utilized for primary major incident triage allowing ALS providers to focus on more clinical roles. 


\section{ABBREVIATIONS}

ALS: $\quad$ Advanced Life Support practitioner

BLS: $\quad$ Basic Life Support practitioner

EMS: $\quad$ Emergency Medical Services

ILS: $\quad$ Intermediate Life Support practitioner

MIMMS: $\quad$ Major Incident Medical Management and Support 


\section{INTRODUCTION}

Major incidents typically have a chaotic aftermath and demand special arrangements to ensure the effective delivery of adequate medical management. ${ }^{(1)}$ Triage is particularly important in these situations where the surge in healthcare demand often outweighs existing resources. Triage is therefore a critical component of major incident medical management; ensuring that the right patient gets to the right facility at the right time. ${ }^{(1)(2)(3)}$

Major incident triage systems differ from routine prehospital triage and is determined by the type and severity of live casualties as well as their number and dispersion within the incident area. $\left({ }^{4}\right)$ Effective triage occurs in two phases, each phase with a different objective. Primary triage occurs at the scene of the incident before immobile patients are moved and is a rapid evaluation to prioritize casualties who need urgent medical care. Examples of primary triage algorithms are START (Simple Treatment and Rapid Transport), STM (Sacco Triage Method), Care Flight Triage and the Triage Sieve. ${ }^{(2)(5)}$ Secondary triage is done at casualty receiving sites at or close to the incident where more time and resources are available for a more in-depth assessment. $^{(5)}$ Algorithms for secondary triage include SAVE (Secondary Assessment of Victim Endpoint) and Triage Sort. ${ }^{(6)(7)}$

Triage algorithms allow for an objective assessment according to pre-set criteria, thereby improving triage accuracy and reliability. ${ }^{(8)}$ However, triage errors do occur resulting in patients being inappropriately classified. These errors subsequently lead to inappropriate resource utilization and essentially hinders the delivery of effective emergency care. ${ }^{(9)(10)}$ Under-triage occurs when victims with life-threatening injuries requiring immediate treatment are incorrectly classified to receive delayed care. On the other hand, over-triaged patients have non-critical injuries but are classified as urgent and thus requiring immediate care. Over triage seems to occur more frequently than under triage with rates documented between $40 \%$ and $89 \%$ compared to less than $15 \%$ of the time..$^{(11)(12)(13)}$ 
An important reason for triage errors is the lack of adherence to the triage algorithm, occurring up to $26 \%$ of the time. ${ }^{(11)(14)(15)}$ Fitzharris et al found variations in triage performance across different training levels of prehospital personnel where the highest adherence rates $(77 \%)$ occurred among the lowest-level trained personnel. ${ }^{(14)}$ Selecting the most appropriate responder to perform triage during a major incident might help to reduce these errors. ${ }^{(16)}$ The aim of this study was to evaluate and compare the triage accuracy between three levels of prehospital emergency care providers in South Africa using the Triage Sieve algorithm.

\section{METHODS}

\section{Study design}

A cross sectional study was done between March and October 2016 after receiving approval by the Health Research Ethics Committee of Stellenbosch University (S15/10/238).

\section{Study setting}

South Africa has a high burden of major incidents, ${ }^{(17)}$ and the management thereof is guided by the 2002 Disaster Management Act. ${ }^{(18)}$ It involves coordinated efforts between the Fire and Rescue Service, the South African Police Service, and Emergency Medical Services (EMS). South Africa adopted the Major Incident Medical Management and Support (MIMMS) principles as part of the Fédération Internationale de Football Association (FIFA) 2010 Soccer World cup legacy. The triage system used is that of Triage Sieve (primary triage) and Triage Sort (secondary triage). ${ }^{(19)}$ Triage Sieve measures various physiological parameters that determine the priority for treatment (Figure 1). Patients are categorized into one of four groups: 1) Red (priority 1) - Patients whose life is in immediate danger and require immediate treatment; 2) Yellow (priority 2) -Patients not in immediate danger, but do require urgent surgical or medical intervention within 2 to 4 hours; 3) Green (priority 3) - Patients with minor injuries; and 4) Blue (no priority) - patients who are either dead or have extensive injuries that 
cannot be saved with the limited resources available. ${ }^{(20)}$ Similar to the United Kingdom, use of the Blue category is controversial in South Africa with an undocumented consensus to exclude it from primary triage categories. ${ }^{(1)}$

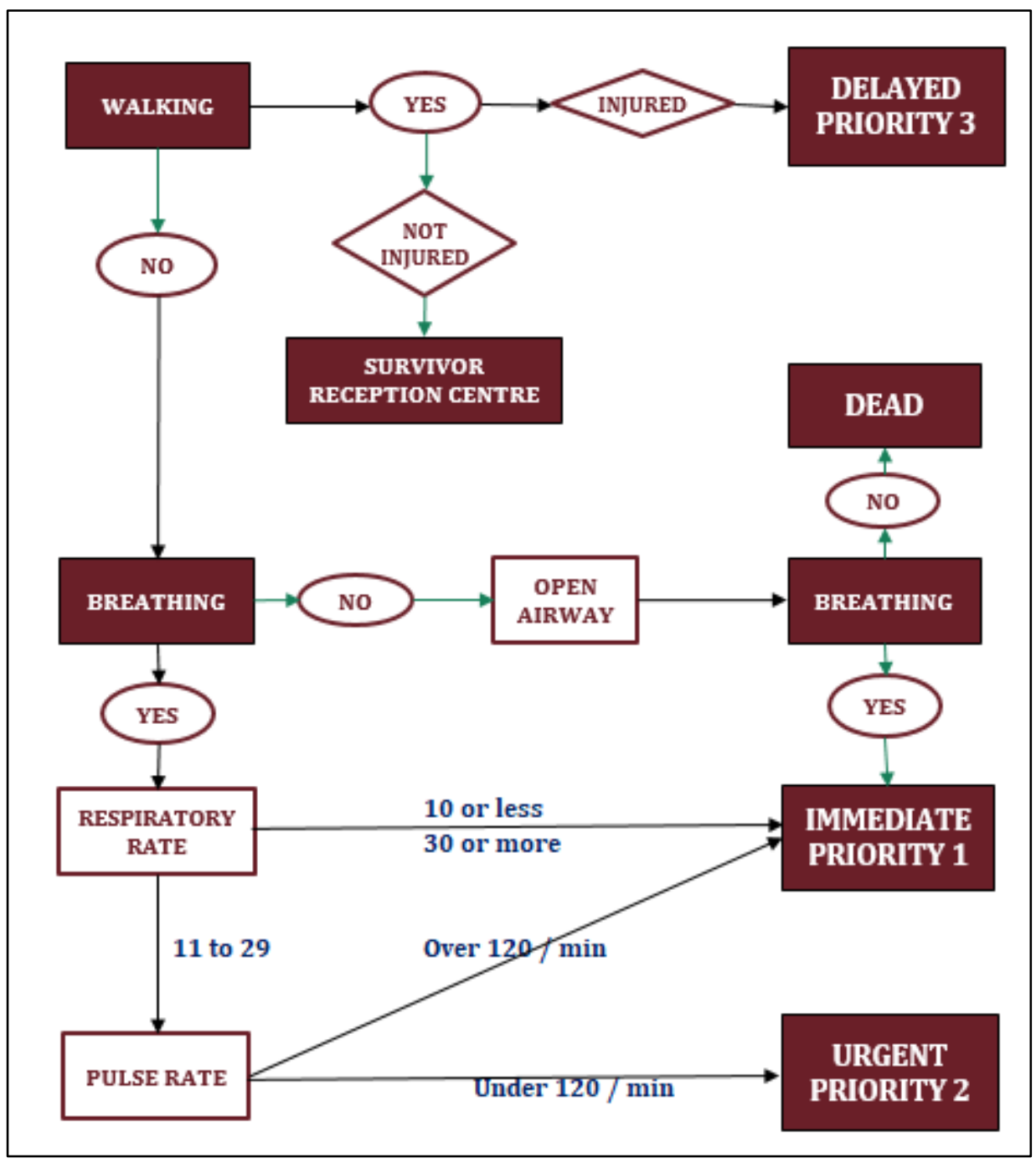

Figure 1: Triage Sieve algorithm

Prehospital healthcare providers in South Africa are divided into three groups according to training levels: Basic Life Support (BLS) practitioners (entry level emergency care providers), Intermediate Life Support (ILS) practitioners (mid-level emergency care providers) and 
Advanced Life Support (ALS) practitioners or paramedics (advanced-level emergency care providers).

\section{Study population}

There are 1,510 prehospital emergency care providers in the Western Cape government health service; 614 BLS, 644 ILS and 252 ALS (Email, H. Hendricks, Manager EMS information Management, $12^{\text {th }}$ February 2018). All providers attending MIMMS training in the Western Cape from March to October 2016 were eligible to participate. The Western Cape is the southernmost of South Africa's nine provinces, covering an area of $129,462 \mathrm{~km}^{2}$ with a population of 6.5 million. ${ }^{(21)}$ It is divided into five rural districts (Cape Winelands, Central Karoo, Eden, Overberg, West Coast), and one metropolitan district (City of Cape Town). ${ }^{(22)}$ Inclusion criteria were prehospital healthcare providers registered with the Health Professions Council of South Africa and practicing in South Africa. Trainers of major incident courses were excluded.

\section{Data collection}

Twenty different multiple-choice questions were created before the start of the study and consisted of structured case scenarios (vignettes) with casualties from a fabricated major incident requiring triage. These questions were validated by an expert panel consisting of five emergency medicine physicians considered to be experts based on their knowledge, training and experience in prehospital medicine in South Africa, and specifically in the use of the Triage Sieve algorithm. The experts independently assigned a triage category to the patient in each vignette. The same triage category (100\% agreement) was assigned in 16 questions. The remaining four questions were subsequently discussed and the correct answer decided by consensus agreement. The validated questions (Appendix 1) were used to determine the triage accuracy. 
All attendees were informed about the study at the start of the MIMMS training. Consented participants were allocated a random identification number before completing a basic demographic questionnaire (Appendix 2) and a pre-training written test consisting of 20 multiple-choice questions.

After the formal major incident training, which included the Triage Sieve algorithm, participants completed a written post-training test (consisting of 20 multiple-choice questions) and a faceto-face evaluation. Additional pressure was placed on the trainee during the face- to-face evaluation. Trainees were individually assessed by one trainer who randomly selected any three of the validated questions and presented it to the trainee as a patient requiring triage. The time from when the scenario was presented to when the trainee allocated a triage category was noted for each of the three selected cases. The Triage Sieve algorithm was displayed during all the tests. The same course trainers were used throughout the data collection period to reduce bias. Providers not participating in the study received the same standard of teaching. The first MIMMS course during the data collection period was used as a pilot study in order to improve the process for the participants, the MIMMS trainers and the data collectors. Data from the pilot study was not included in the data analysis.

Two outcomes were measured to assess participants' mass casualty triage performance: i) triage accuracy, and ii) duration of triage. Triage accuracy was measured by comparing the participant's answer to the validated answers of the expert group. The results are presented as percentage of 'correct' triage, over triage and under triage. The duration of triage was considered the total time for completing triage during the face-to-face evaluation. 


\section{Data management}

Test sheets were marked by the lead investigator (AAN). A second examiner who had no medical training and who did not participate in any other part of the study crosschecked the marks. Data were subsequently cleaned, entered into a Microsoft Excel spreadsheet Version 2013 (Microsoft Corporation, Redmond, Washington, USA) and then exported for analysis to STATA 14 (StataCorp. 2015, College Station, Texas, USA). Data entering was again checked for completion by the second examiner. Data were kept in an access-controlled location.

\section{Data analysis}

Standard descriptive statistics are presented. Means and standard deviations or medians and interquartile ranges were used to describe continuous variables. Categorical data are described using frequencies or percentage with $95 \%$ confidence intervals $(\mathrm{CI})$, where appropriate. Chisquare and Fisher exact tests were used to compare basic demographics between groups. For the primary outcome (overall accuracy scores), one way ANOVA or logistic regression, where appropriate, was performed to determine significant differences between providers. A 5\% significance level was applied. Normality was checked both quantitatively and qualitatively.

\section{RESULTS}

136 participants attended the MIMMS during the data collection period and all were recruited for the study. Only data from 129 participants were analyzed after seven were excluded (Figure 2). 


\section{Enrolment}

\section{Assessment}

\section{Analysis}

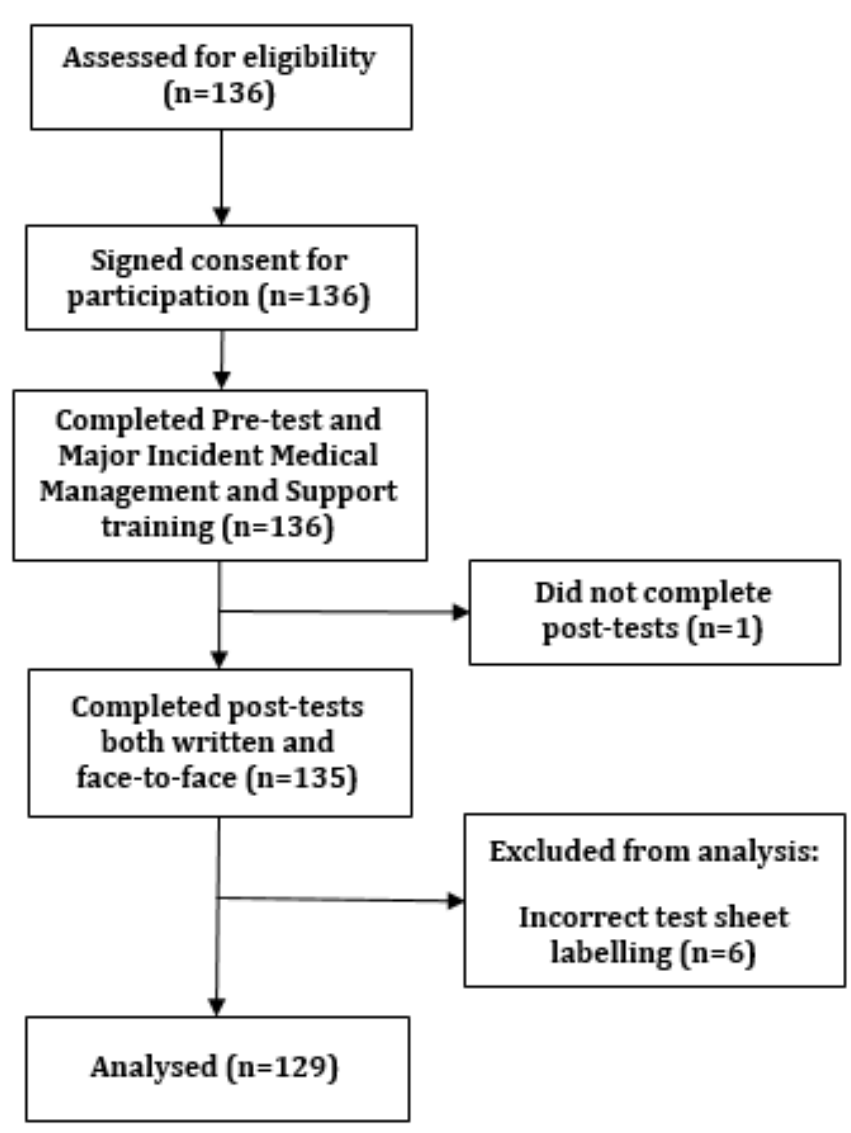

Figure 2. Flow chart of the study population

Study participants had a similar gender distribution (male $n=65,50.39 \%$ ) with a mean age of 33.4 years (standard deviation (SD) 7.77 years). Most of the participants $(n=87,67.44 \%)$ were BLS providers. A total of $72(55.81 \%)$ participants were working in the rural areas of the Western Cape, while 80 (62.02\%) participants had worked for the provincial government EMS for less than 5 years. Only $41(31.78 \%)$ of the participants reported having prior major incident training including triage sieve protocol while 17 (13.18\%) of these having been trained 5 years or more before the study (Table 1).

The overall correct triage score pre-training was $53.91 \%$ (95\% CI 51.98 to 55.83), over triage $31.43 \%$ (95\% CI 29.66 to 33.2) and under triage $13.84 \%$ (95\% CI 12.55 to 12.22 ). After training, the overall correct triage score increased to $63.60 \%$ (95\% CI 61.72 to 65.44 ), ober 
triage decreased to $17.91 \%$ (95\% CI 16.47 to 19.43 ) and under triage increased to 17.83 (95\% CI 16.40 to 19.36) (Figure 3).

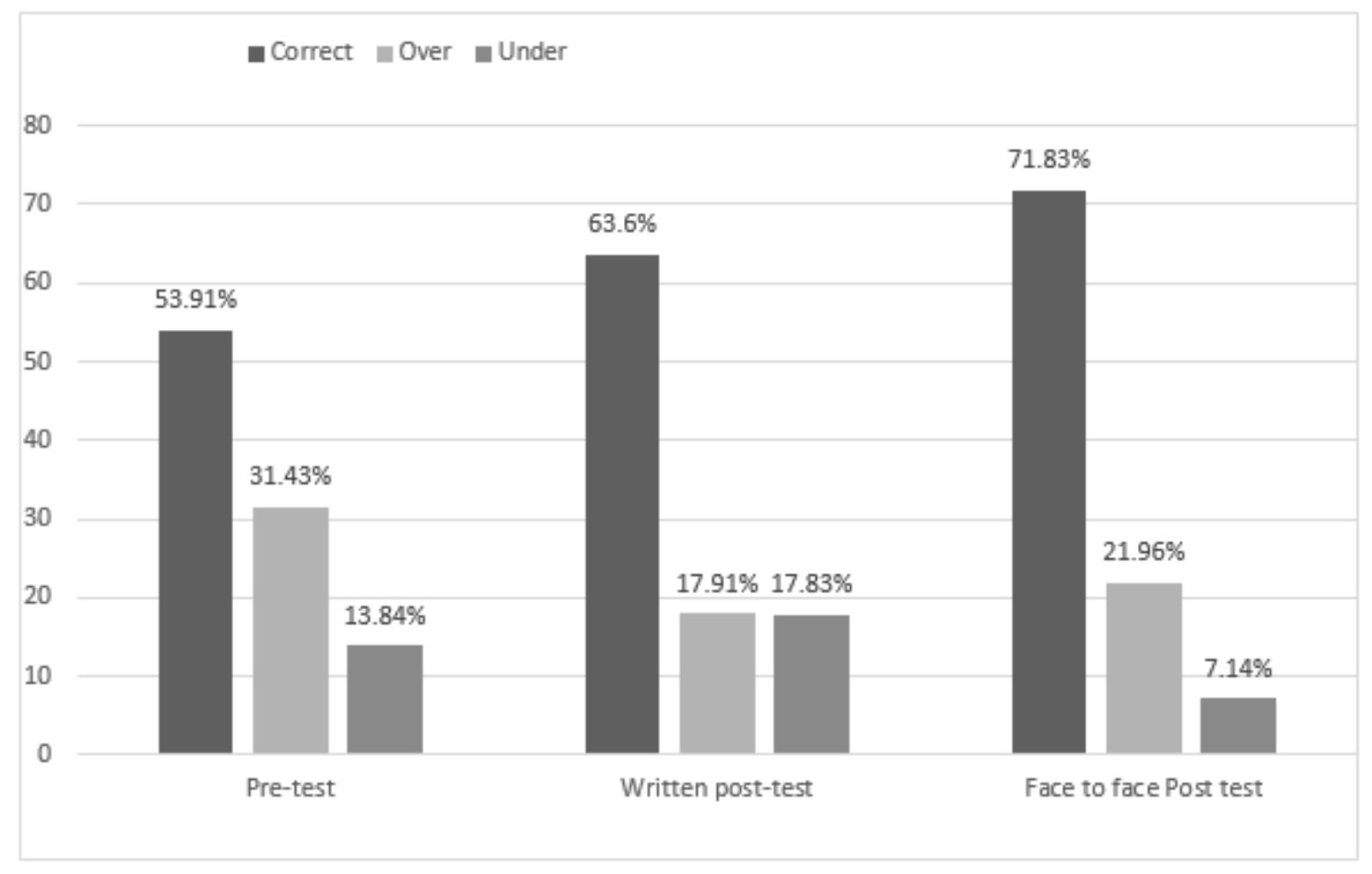

Figure 3: Triage accuracy for all study participants using the Triage Sieve algorithm

ALS providers had the highest percentage of correct triage scores both pre and post-training, with the lowest rates of over and under triage (Table 2).

There was no difference in triage scores across the different age groups $(\mathrm{p}=0.229)$. However, participants who previously received major incident training performed better compared to those with no prior training $(\mathrm{p}=0.001)$.

The duration of triage (median, interquartile range) was the shortest for ALS providers ( $3 \mathrm{sec}$, 2 to $7 \mathrm{sec}$ ) followed by BLS (4 sec, 2 to $8 \mathrm{sec})$ and ILS ( $5 \mathrm{sec}, 3$ to $9 \mathrm{sec})(\mathrm{p}=0.034)$. 


\section{DISCUSSION}

This is the first study to compare triage accuracy and duration between different levels of prehospital healthcare providers in South Africa using the Triage Sieve algorithm. The overall correct score post major incident training was a mere $64 \%$. There were no significant differences in triage accuracy between the different levels of providers after training, although ALS providers were more likely to perform the triage correct (odds ratio 1.21). The duration of triage was also not clinically significant between the different levels of providers. Previous major incident training made a substantial difference in triage performance $(\mathrm{p}=0.001)$.

The overall correct triage score of $64 \%$ across all levels of prehospital providers is disconcertingly low. Risavi et al found scores of $75 \%$ across all levels of paramedics, another study found $84 \%$ accuracy amongst paramedics ${ }^{(23)}$ while another found $79.9 \%$ for primary care paramedic trainees and $72.0 \%$ for Fire Service trainees. ${ }^{(24)}$ However, a $10 \%$ improvement in triage scores was noted after providers underwent the MIMMS training. This trend of improvement post training is similar to findings in other studies. Sapp et al described that first year medical students who received brief START training had comparable triage performance to qualified nurses, paramedics and emergency physicians. ${ }^{(25)}$ Another study found similar triage scores in primary care paramedic and fire science students after training. Despite this trend towards improvement, the low correct scores are concerning for a country with high numbers of major incidents. ${ }^{(17)}$

Triage error rates varied from previous studies. Over triage rate was similarly $<50 \%$ while under triage rate was 3.6 times higher than the recommended $<5 \%$. $^{(26)(27)}$ Other studies show diverse findings with no noticeable trend. In a Dutch study, under triage occurred in $10.9 \%$ (95\% CI 7.4 to 15.7 ) of cases with over triage being $39.5 \%$ (95\% CI 36.9 to 42.1$).{ }^{(11)}$ While in a retrospective study of the Turkish Airline crash, of the 135 victims triaged by ambulance teams, there was an over triage rate of $89 \%$ and under triage rate of $12 \% .^{(12)(13)}$ 
When comparisons were made between the various qualifications, ALS providers had the highest correct triage scores with the lowest over triage rates. This is inconsistent with other studies which showed better performance and algorithm adherence rates by the least qualified levels of prehospital providers compared to the most advanced. Fitzharris et al in their New South Wales protocol adherence study found variations in performance across the different levels of prehospital personnel with highest adherence rates (77\%) among the lowest-level trained personnel. ${ }^{(14)}$ A Dutch study on protocol adherence by EMS personnel in triaging 1,607 victims of high energy impact trauma found adherence rate of 78.7\% (310 patients were not transported to the required level trauma center). ${ }^{(11)}$ Similar findings of protocol non-adherence were reported by Wong et al in their Hong Kong study assessing appropriate diversion of 141 major trauma cases by paramedics. Over-diversion rate was $3.5 \%$ with under-diversion at $40.5 \%$; overall accuracy was $74.5 \% .^{(15)}$

There were no substantial differences in triage scores between different provider levels after receiving formal major incident training. It is interesting to note that while they generally performed better, there was no significant improvement in the triage accuracy by the ALS providers pre and post training (65\% to 68\%) compared to both ILS (53\% to 63\%) and BLS (51\% to $63 \%)$. The reason for this was not explored.

Prior major incident training was found to be an independent factor contributing to better performance. This was not the case in similar studies amongst firemen and other prehospital providers where prior training did not make any statistical difference. ${ }^{(28)(29)}$

\section{Limitations}

The study had several limitations potentially influencing the generalizability of the results. Firstly, the tests were completed in a controlled environment. While the post-training face-toface evaluation was introduced to simulate additional pressure, it still lacks the multiple external factors and realism of a real major incident. Secondly, major incident training is not compulsory for all prehospital personnel. This introduced selection bias as participants interested in major 
incidents were most likely to attend the MIMMS course. Findings might therefore not reflect the entire EMS system. Lastly, only one province was included and care should be taken in extrapolating the results to the rest of South Africa or other EMS systems.

\section{Suggestions for future research}

Prior major incident training was an important factor and more research is needed to determine adequate follow-up training. The triage performance of non-healthcare personnel that would respond to major incidents (e.g. fire, traffic, and police) should also be evaluated to determine whether they might be more suitable to perform primary triage.

\section{CONCLUSION}

The ability of all levels of prehospital emergency care providers in South Africa's state owned EMS system to correctly use the Triage Sieve algorithm is less than optimal. Although accuracy improved after formal major incident training, there was no substantial difference between the three provider levels. ALS providers performed the triage quicker than the other groups, but this difference was not clinically significant. In the local environment, BLS and ILS providers with major incident training can thus be utilized for primary major incident triage allowing ALS providers to take on more clinically orientated roles. Prior major incident training made a significant difference in triage accuracy. 


\section{REFERENCES}

1. Lax P, Prior K. Major incident pre-hospital care. Surgery (Oxford), Elsevier Ltd; 2015; 33 (9): 419-423

2. Baker MS. Creating order from chaos: part I: triage, initial care, and tactical considerations in mass casualty and disaster response. Mil Med. 2007; 172(3):232-6.

3. Vassallo SLJ, Horne LCS, Ball S, Whitley LJ. UK Triage the validation of a new tool to counter an evolving threat. Injury, Elsevier Ltd; 2014;45(12):2071-5.

4. Bostick NA, Subbarao I, Burkle FMJ, Hsu EB, Armstrong JH, James JJ. Disaster triage systems for large-scale catastrophic events. Disaster Med Public Health Prep. United States; 2008 Sep;2 Suppl 1:S35-9.

5. Jenkins JL, McCarthy ML, Sauer LM, Green GB, Stuart S, Thomas TL, et al. Masscasualty triage: time for an evidence-based approach. Prehospital disaster Med 2008;23(1):38.

6. Helenis M. Major incident medical management and support: The practical approach at the scene. Emerg Nurse. RCN Publishing Ltd; 2012;20(3):9.

7. Benson M, Koenig KL, Schultz CH. Disaster triage: START, then SAVE--a new method of dynamic triage for victims of a catastrophic earthquake. Prehosp Disaster Med. 1996;11(2):117-24.

8. Martí R, Robles S, Martín-Campillo a., Cucurull J. Providing early resource allocation during emergencies: The mobile triage tag. J Netw Comput Appl. 2009;32(6):1167-82.

9. Frykberg ER. Triage: Principles and practice. Scand J Surg. 2005;94(4):272-8.

10. Frykberg ER. Medical management of disasters and mass casualties from terrorist bombings: how can we cope? J Trauma. United States; 2002 Aug;53(2):201-12. 
11. Van Laarhoven JJEM, Lansink KWW, Van Heijl M, Lichtveld R a., Leenen LPH. Accuracy of the field triage protocol in selecting severely injured patients after high energy trauma. Injury; 2014;45(5):869-73.

12. Postma ILE, Weel H, Heetveld MJ, Van Der Zande I, Bijlsma TS, Bloemers FW, et al. Patient distribution in a mass casualty event of an airplane crash. Injury; 2013;44(11):1574-8.

13. Postma ILE, Weel H, Heetveld MJ, Dan Der Zande I, Bijlsma TS, Bloemers FW, et al. Mass casualty triage after an airplane crash near amsterdam. Injury; 2013;44(8):1061-7.

14. Fitzharris M, Stevenson M, Middleton P, Sinclair G. Adherence with the pre-hospital triage protocol in the transport of injured patients in an urban setting. Injury; 2012;43(9):136876.

15. Wong C, Lui C, So F, Tsui K TS. Prevalence and predictors of under-diversion in the primary trauma diversion system in Hong Kong. Hong Kong J Emerg Med. September 2013 20(5):276-286

16. Robertson-Steel I. Evolution of triage systems. Emergency Medicine Journal : EMJ. 2006; 23(2):154-155.

17. Van Hoving D, Lategan H, Wallis L, Smith W. The epidemiology of major incidents in the Western Cape Province, South African Medical Journal, vol. 105, issue 10 (2015) p. 831

18. Annual report 1 April 2016 to 31 March 2017 provincial disaster management centre. Cape Town; 2017, https://www.westerncape.gov.za/sites/www.westerncape.gov.za/files/wcdmc__annual_report_16-17-_signed_copy.pdf (accessed 4th November 2017)

19. Mackway-Jones K. Major Incident Medical Management and Support: The Practical Approach at the Scene. In Wiley-Blackwell; 2011.p. 89-102. 
20. Smith W. Triage in mass casualty situations. CME : Your SA Journal of CPD, Volume 30, Issue 11, Nov 2012, p. 413 - 415

21. Statitistics South Africa Mid-year population estimates. Pretoria July 2017; http://www.statssa.gov.za/publications/P0302/P03022017.pdf (accessed $4^{\text {th }}$ November 2017)

22. Statistics South Africa. Census 2011 Municipal Report - Western Cape. Pretoria: SSA, 2012. https:// www.statssa.gov.za/Census2011/Products/WC_Municipal_Report.pdf (accessed 4 September 2014).

23. Deluhery MR, Lerner EB, Pirrallo RG, Schwartz RB. Paramedic Accuracy Using SALT Triage After a Brief Initial Training. Prehospital Emerg Care. 2011 Oct-Dec; 15 (4):52632

24. Lee CWC, McLeod SL, Van Aarsen K, Klingel M, Franc JM, Peddle MB. First Responder Accuracy Using SALT during Mass-casualty Incident Simulation. Prehosp Disaster Med.2016Apr;31(2):150-4.

25. SappRF, Brice JH, Myers JB et al. Triage performance of first-yearmedical students using a multiple-casualty scenario, paper exercise. Prehosp DisasterMed. 2010;25(3):239-45.

26. Challen K WD. Major incident triage: comparative validation using data from 7th July bombings. Injury. 2013;44(5):629-33.

27. Carron PN, Taffe P, Ribordy V et al. Accuracy of prehospital triage of trauma patients by emergency physicians: a retrospective study in western Switzerland. Eur J Emerg Med. 2011;18(2):(86-93).

28. Kilner T HF. Triage decisions of United Kingdom police firearms officers using a multiple-casualty scenario paper exercise. Prehosp Disaster Med. 2005;20(1):40-46. 
29. Nilsson, Abraham et al. "Improved and Sustained Triage Skills in Firemen after a Short Training Intervention." Scandinavian Journal of Trauma, Resuscitation and Emergency Medicine 23(2015):81.

30. Risavi BL1, Salen PN, Heller MB AS. A two-hour intervention using START improves prehospital triage of mass casualty incidents. Prehosp Emerg Care. 2001;(5(2)):197-9. 


\section{APPENDICES}

\section{Appendix 1: Major Incident Triage Test}

Study Number:

Date:

Training Venue:

Please read the scenario below before answering the questions

You are part of the first ambulance team responding to a major incident where a bus and taxi had collided. The incident occurred on a busy highway and 10 additional vehicles were subsequently involved in the collision. You are tasked to rapidly assess and triage the involved victims.

Using the Triage Sieve algorithm (supplied) for primary triage in major incidents, please indicate the appropriate triage category for the following 20 victims:

1. The driver of the taxi is standing near his wrecked taxi, extremely anxious. He walks towards you and demand the police. You notice a bone protruding through a minimally bleeding wound on his forearm. You do a quick assessment: Blood pressure $=$ 100/60mmHg, Heart rate $=120 /$ minute, Respiratory rate $=26 /$ minute .

\begin{tabular}{|l|l|r|r|r|r|}
\hline Triage & & Red & Yellow & Green & Blue \\
Category: & & & & & \\
\hline
\end{tabular}

2. A middle aged man has no obvious injuries. He is lying unconscious with a respiratory rate of 8 breaths / minute and a heart rate of 110 / minute.

\begin{tabular}{|l|r|r|r|r|}
\hline Triage Category: & Red & Yellow & Green & Blue \\
\hline
\end{tabular}


3. A middle aged man runs towards you screaming for help. His shirt and trousers are stained with blood. He is pressing his left palm over the side of his neck. He removes his hand and a jet of blood shoots from his neck. You ask him to continue applying pressure while you check his vital signs. Blood pressure 110/70mmHg, Respiratory Rate: 28/min, Heart Rate: 130/min.

\begin{tabular}{|l|r|r|r|r|}
\hline Triage Category: & Red & Yellow & Green & Blue \\
\hline
\end{tabular}

4. A 10 year old child's foot is pinned under the wheel of one of the vehicles. He is talking to you but is in a lot of pain. You count a respiratory rate of 11 breaths per minute and a thready rapid pulse of 103 beats/minute.

\begin{tabular}{|l|r|r|r|r|}
\hline Triage Category: & Red & Yellow & Green & Blue \\
\hline
\end{tabular}

5. A 26 year old man was ejected him from his car after it caught fire and exploded. You find him 15 meters away from the burning vehicle. He has burn wounds all over his body except for the dorsal surfaces of his feet. The hair on his head and nostrils is charred. He is fully conscious and talks to you but he cannot walk or see. His only complaint is severe pain from the burns around his face. His blood pressure is $76 / 40$ mmHg, Heart rate: 140 bpm, and Respiratory rate: 40 bpm.

\begin{tabular}{|l|r|r|r|r|}
\hline Triage Category: & Red & Yellow & Green & Blue \\
\hline
\end{tabular}


6. The mother of the child in the above question $(\mathrm{Q} 4)$ is lying away from her son. She responds weakly to your calls and occasionally moans in pain. A loose piece of metal has transected through her right foot and a small pool of blood has collected around her. She has a weak rapid pulse of 150 beats/minute and Respiratory Rate of 27 breaths/minute.

\begin{tabular}{|l|r|r|r|r|}
\hline Triage Category: & Red & Yellow & Green & Blue \\
\hline
\end{tabular}

7. A young woman, with a bleeding wound on her face is still strapped in the driver's seat in one of the vehicles. She responds to your voice and instructions. Her respiratory rate is 18 breaths/minute and her heart rate is 120 beats/minute. You open the car door, undo the seat belt and ask her to step out. She is able to walk unaided.

\begin{tabular}{|l|r|r|r|r|}
\hline Triage Category: & Red & Yellow & Green & Blue \\
\hline
\end{tabular}

8. A middle aged woman has deep circumferential burns involving her entire right upper limb. She is also complaining of mild headache and cannot walk. Her vital signs are: Respiratory rate 23 breaths/minute Heart rate: 100 beats/minute, and Blood pressure: $102 / 72 \mathrm{mmHg}$.

\begin{tabular}{|l|r|r|r|r|}
\hline Triage Category: & Red & Yellow & Green & Blue \\
\hline
\end{tabular}

9. An elderly man was jogging by the roadside before being hit by a vehicle. He is lying unconscious in a small pool of blood. He has feeble chest movements and you calculate a respiratory rate of 7 breaths/ minute with a Heart rate of 140 beats/minute.

\begin{tabular}{|l|r|r|r|r|}
\hline Triage Category: & Red & Yellow & Green & Blue \\
\hline
\end{tabular}


10. A 14 year old is crying while seated with her back against a tree next to the road. She cannot stand or walk. There's minor bruising over her abdomen (left upper quadrant). She is breathing at a rate of 20 breaths/minute and her heart rate is 100 beats per minute.

\begin{tabular}{|l|r|r|r|r|}
\hline Triage Category: & Red & Yellow & Green & Blue \\
\hline
\end{tabular}

11. An elderly woman is walking around her wrecked car in continuous circles. She is mumbling to herself and is obviously confused. Her left wrist is injured and deformed. There are no other apparent injuries. Heart Rate: 110 Beats/minute, Respiratory Rate: 20 breaths/ minute.

\begin{tabular}{|l|r|r|r|r|}
\hline Triage Category: & Red & Yellow & Green & Blue \\
\hline
\end{tabular}

12. A pregnant woman is lying in the middle of the road. She is alert but cannot stand as her left lower leg (tibia) is fractured and protruding through the skin. Her respiratory rate is 28 beats/minute and heart rate is 140 beats/minute.

\begin{tabular}{|l|r|r|r|r|}
\hline Triage Category: & Red & Yellow & Green & Blue \\
\hline
\end{tabular}

13. A young woman's head scarf is bloody and she is bleeding through her left ear and left nostril. She is alert, oriented and able to speak to you. She can walk but has a severe headache. Her respiratory rate is 20 breaths/minute and her heart rate 118 beats/minute.

\begin{tabular}{|l|r|r|r|r|}
\hline Triage Category: & Red & Yellow & Green & Blue \\
\hline
\end{tabular}


14. A 17 year old male, in obvious respiratory distress, is lying on the pavement, unable to stand or walk. There is minor bleeding from his left knee. His respiratory rate is 25 breaths/minute, rapid and shallow. His heart rate 110 beats/min.

\begin{tabular}{|l|r|r|r|r|}
\hline Triage Category: & Red & Yellow & Green & Blue \\
\hline
\end{tabular}

15. An elderly woman is lying unconscious next to her vehicle. There's a wheelchair attached to the roof. You cannot see any chest movements. You open her mouth and find a set of loose dentures in her mouth. You remove them and open her airway with a jaw thrust maneuver. She starts to breath spontaneously at 14 breaths/minute. Her heart rate is 98 beats/minute.

\begin{tabular}{|l|r|r|r|r|}
\hline Triage Category: & Red & Yellow & Green & Blue \\
\hline
\end{tabular}

16. The drunk bus driver was punched by one of his angry passengers. He fell and hit his head against the pavement. He is conscious but cannot stand or walk. His heart rate is 120 beats/minute and respiratory rate 9 breaths/minute.

\begin{tabular}{|l|r|r|r|r|}
\hline Triage Category: & Red & Yellow & Green & Blue \\
\hline
\end{tabular}

17. A 19 year old girl didn't wear a seat belt and the impact of the injury pushed her through the car's windscreen. Both her lower legs (tibias) are broken and she has multiple cut wounds her face and hands. Some wounds have pieces of glass in them. She is fully conscious, with a respiratory rate of 12 breaths/minute and a heart rate of 110 beats/minute.

\begin{tabular}{|l|r|r|r|r|}
\hline Triage Category: & Red & Yellow & Green & Blue \\
\hline
\end{tabular}


18. One of the drivers are still stuck in his car. A huge metallic pipe (about 10 centimeter in diameter) has pierced through his back and is protruding through the right side of his chest. He is unresponsive, with shallow breaths at 10 breaths/minute. Both his arms have been severed at the elbows. He has a weak carotid pulse of 120 beats/minute.

\begin{tabular}{|l|r|r|r|r|}
\hline Triage Category: & Red & Yellow & Green & Blue \\
\hline
\end{tabular}

19. A 24 year old man runs towards you, howling in pain!! He is holding his left shoulder which appears to be dislocated. His heart rate is 122 beats/minute and his respiratory rate is 23 breaths/minute.

\begin{tabular}{|l|r|r|r|r|}
\hline Triage Category: & Red & Yellow & Green & Blue \\
\hline
\end{tabular}

20. A 12 year old girl is lying in the road, eyes closed. She responds to painful stimulation but is unable to stand or walk. She is wearing a costume for a school play, which has turned bloody. You notice blood oozing from at her left wrist. Her pulse rate is 132 beats/minute with a respiratory rate of 16 breaths/minute.

\begin{tabular}{|l|r|r|r|r|}
\hline Triage Category: & Red & Yellow & Green & Blue \\
\hline
\end{tabular}




\section{Appendix 2: Survey Tool}

A COMPARISON BETWEEN DIFFERENTLY SKILLED PREHOSPITAL EMERGENCY

CARE PROVIDERS IN MASS CASUALTY TRIAGE IN SOUTH AFRICA

Study number:

Training Site:

Date:

SECTION A: DEMOGRAPHICS: (TO BE COMPLETED BY PARTICIPANTS)

\begin{tabular}{|l|l|l|l|l|l|l|}
\hline Age:_(Years) & \multicolumn{5}{|l|}{} \\
\hline Sex: $\underline{\text { tick) }}$ & \multicolumn{2}{|l|}{ MALE } & \multicolumn{2}{l|}{ FEMALE } \\
\hline $\begin{array}{l}\text { Duration of Service } \\
\text { (in years): }\end{array}$ & $<1$ & $1-2$ & $2-3$ & $3-4$ & & $>5$ \\
\hline
\end{tabular}

SECTION B: TRAINING:

\begin{tabular}{|l|l|l|l|}
\hline & BLS & ILS & ALS \\
\hline Level of Training (tick): & & & \\
\hline Year Completed & & & \\
& & & \\
\hline
\end{tabular}

\section{Prior Major Incident Training:}

\begin{tabular}{|l|l|l|}
\hline Have you had Prior Major Incident Management & YES & NO \\
training? & Where & When ( Year) \\
\hline If YES, state details & & \\
\hline
\end{tabular}


SECTION C: (TO BE COMPLETED BY INVESTIGATORS)

PHASE 1: PRE-TEST

\begin{tabular}{|l|l|l|}
\hline Correct Triage & Over Triage & Under triage \\
\hline & & \\
& & \\
\hline
\end{tabular}

\section{PHASE 2:}

\begin{tabular}{|l|l|l|}
\hline MIMMS training completed: & Yes & No \\
& & \\
\hline
\end{tabular}

PHASE 3: POST-TEST

WRITTEN:

\begin{tabular}{|l|l|l|}
\hline Correct Triage & Over Triage & Under triage \\
\hline & & \\
\hline
\end{tabular}

FACE-TO-FACE:

\begin{tabular}{|l|l|l|l|}
\hline Correct Triage & Over Triage & Under triage & Time taken to \\
& & & \\
\hline & & & \\
\hline & & & \\
\hline
\end{tabular}




\section{Appendix 3: Table 1}

Table 1. Demographics of participants divided according to training level

\begin{tabular}{|c|c|c|c|c|}
\hline & $\begin{array}{l}\text { BLS } \\
\mathrm{n}(\%)\end{array}$ & $\begin{array}{l}\text { ILS } \\
\mathrm{n}(\%)\end{array}$ & $\begin{array}{l}\text { ALS } \\
\mathrm{n}(\%)\end{array}$ & $\begin{array}{l}\text { TOTAL } \\
\text { n }(\%)\end{array}$ \\
\hline Total & $87(67.44)$ & $22(7.05)$ & $20(15.50)$ & $129(100)$ \\
\hline \multicolumn{5}{|l|}{ Gender } \\
\hline Male & $43(33.33)$ & $12(9.30)$ & $10(7.75)$ & $65(50.39)$ \\
\hline Female & $44(34.11)$ & $10(7.75)$ & $10(7.75)$ & $64(49.61)$ \\
\hline Age: mean (SD) & $33.58(6.94)$ & $33.05(8.24)$ & $33(7.58)$ & $33.14(7.77)$ \\
\hline \multicolumn{5}{|l|}{ Area of service } \\
\hline Rural & $53(41.09)$ & $11(8.53)$ & $8(6.20)$ & $72(55.81)$ \\
\hline Urban & $31(24.03)$ & $10(7.75)$ & $7(5.43)$ & $48(37.21)$ \\
\hline Did not answer & & & & $9(6.98)$ \\
\hline \multicolumn{5}{|c|}{ Duration of service } \\
\hline$<5$ years & $61(47.29)$ & $12(9.30)$ & $7(5.43)$ & $80(62.02)$ \\
\hline$\geq 5$ years & $24(18.60)$ & $10(7.75)$ & $13(10.08)$ & $47(36.43)$ \\
\hline Did not answer & & & & $2(1.55)$ \\
\hline \multicolumn{5}{|c|}{ Prior major incident training } \\
\hline$<5$ years & $12(9.31)$ & $8(6.21)$ & $4(3.10)$ & $24(18.6)$ \\
\hline$\geq 5$ years & $9(6.98)$ & $2(1.55)$ & $6(4.65)$ & $17(13.18)$ \\
\hline Not trained & $64(49.61)$ & $10(7.75)$ & $8(6.20)$ & $82(63.57)$ \\
\hline Did not answer & & & & $6(4.65)$ \\
\hline
\end{tabular}

BLS=Basic Life Support, ILS=Intermediate Life Support, ALS=Advanced Life Support, $\mathrm{SD}=$ Standard Deviation 


\section{Appendix 4: Table 2}

Table 2. Comparison of written triage scores between differently trained prehospital healthcare providers using the Triage Sieve algorithm

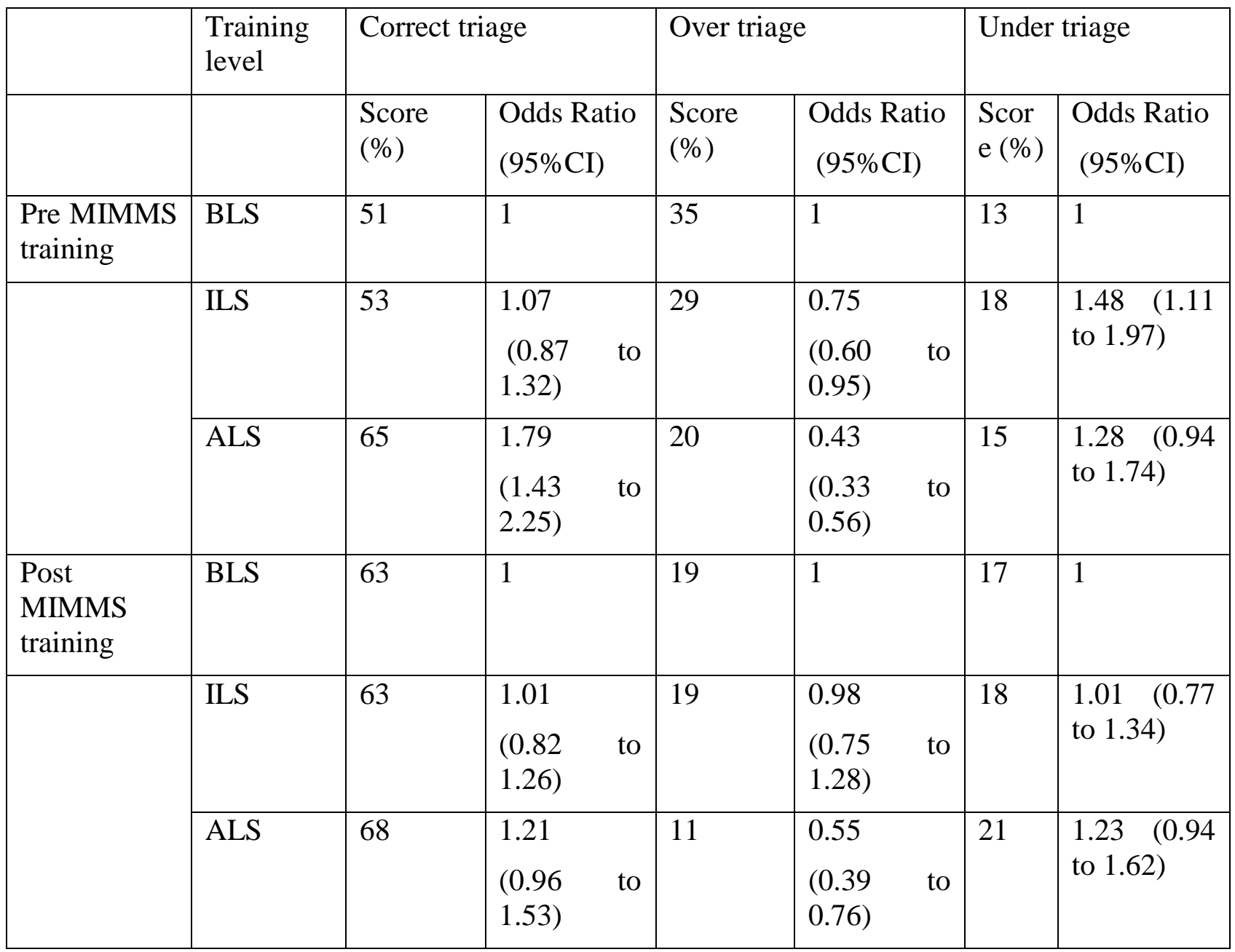

95\% CI $=95 \%$ Confidence Interval, MIMMS= Major Incident Medical Management and

Support, BLS=Basic Life Support, ILS=Intermediate Life Support, ALS=Advanced Life

Support 


\section{PART C: SUPPORTING DOCUMENTATION}




\title{
1) PROPOSAL
}

A COMPARISON BETWEEN DIFFERENTLY SKILLED PREHOSPITAL EMERGENCY CARE PRACTITIONERS IN MASS CASUALTY TRIAGE IN SOUTH AFRICA

Student:

\author{
ANNET ALENYO NGABIRANO \\ MBChB (MUST), MSc Dis.Med (EMDM) \\ DIVISION OF EMERGENCY MEDICINE \\ STELLENBOSCH UNIVERSITY
}

Supervisors:

DR. DJ VAN HOVING

MBChB, DipPEC (SA), MMed (EmMed), MScMedSci (ClinEpi)

DIVISION OF EMERGENCY MEDICINE

STELLENBOSCH UNIVERSITY

DR. WP SMITH

BSc, MB ChB, EMDM, FCEM (SA)

DIVISION OF EMERGENCY MEDICINE

UNIVERSITY OF CAPE TOWN 


\section{DEFINITION OF KEY TERMS}

Advanced Life Support (ALS) practitioner: The most advanced-level prehospital emergency care practitioner. This includes Critical Care Assistants, Emergency Care Practitioners, Emergency Care Technicians and practitioners in possession of the National Diploma: Emergency Medical Care.

Basic Life Support (BLS) practitioner: An entry level qualification into the pre-hospital emergency care profession. Providers are equipped with the basic skills needed to function within the Emergency Medical Services (EMS) environment. This includes Basic Ambulance Assistants.

Disaster: An uncompensated major incident where the healthcare system is unable to cope despite additional resources.

Disaster Medicine: The prevention, immediate response and rehabilitation of the health problems arising from a disaster.

Intermediate Life Support (ILS) practitioner: A mid-level prehospital emergency care provider. This includes Ambulance Emergency Assistants and Operational Emergency Care Orderlies.

Major Incident: 'Any incident where the location, number, severity or type of live casualties requires extraordinary resources' ${ }^{[1]}$ Can be further classified into: (i) natural or man-made; (ii) simple or compound (infrastructure is lost); and (iii) compensated or uncompensated (healthcare system unable to cope despite additional resources)

Triage: The process of determining the priority of patients' treatments based on the severity of their condition. ${ }^{[2]}$ 


\begin{abstract}
Background: Triage accuracy in major incident management is critically important to ensure proper patient management, appropriate referral and utilisation of resources. Prehospital emergency care practitioners are usually the first medical professionals to arrive at any major incident, and are therefore often task to do primary triage. Specific triage protocols are available for objective assessment of patients but lack of adherence to the triage algorithm frequently occurs.
\end{abstract}

Objective: To determine the difference in mass casualty triage performance between differently skilled pre-hospital emergency care practitioners.

Methods: A cross sectional study will be done from February 2016 to July 2016 during routine major incident training within the Western Cape. All prehospital emergency care practitioners (BLS, ILS, ALS levels) will be eligible except those with experience as trainers in disaster management courses. Part 1 involves creating and validating multiple-choice questions to test the triage accuracy using the triage sieve algorithm. Part 2 takes place during the different training sessions. Consented participants will 1) Complete a written pre-test (20 randomly selected multiple-choice questions); 2) Undergo the formal major incident training (MIMMS); and 3) Complete two post-tests; a written test similar to the pre-test (i.e. 20 multiple-choice questions) and a face-to-face evaluation with a trainer using standard MIMMS triage cards and table-top scenarios where additional pressure is placed on participants. To reduce bias, course trainers will be consistent throughout the data collection period. Practitioners not participating in the study will receive the same standard of teaching. Two outcomes will further be measured: Triage accuracy and duration of triage. Comparison will be made between the different prehospital providers in using the triage sieve protocol relating to the specified outcomes. The effect of participant's gender, age, training and duration of service on triage accuracy will also be determined. 
Conclusion: The investigators anticipate making recommendations for the most reliable workforce to do triage during major incidents. This will reduce errors in triage and potentially result in better resource utilization by early initiation of appropriate treatment and transport to referral sites. 


\section{INTRODUCTION}

\section{Background}

The word "triage", derived from the French verb "tier" meaning sort or sieve has greatly evolved from its $14^{\text {th }}$ Century use to more complex applications today. From grading agricultural products according to quality and price, triage has become a tool for prioritizing casualties for medical care. The earliest documentation of triage for medical use was around 1792 by the Napoleonic surgeons Dominique Jean Larrey and Francois Percy. They applied this principle of sorting to war casualties, providing initial field treatment to injured soldiers prior to transport to hospitals on horse drawn ambulances. ${ }^{[3]}$ This principle remains particularly important in major incidents where the demand for medical care outweighs the existing resources. In these situations, triage is a critical management tool for achieving the key objective of major incident management which is to do the best for the most. ${ }^{[4-6]}$

Major incident events in the past 30 years, repeatedly emphasize the importance of adequate major incident preparation which includes regular training for health personnel to ensure satisfactory performance in their roles when major incidents do occur. ${ }^{[5]}$ Prehospital triage in major incidents is perhaps one of the single most important activities that sets the basis for appropriate treatment and referral of victims. ${ }^{[4-6]}$ The actual practice of major incident triage differs from routine prehospital triage and is determined by the type and severity of injuries and the number and distribution of victims. ${ }^{[7]}$ There are two different types of triage in major incidents; primary and secondary triage which have different objectives. Primary triage is carried out at the site of the incident to prioritize patients in the field for evacuation and transport to definitive medical care; major incident primary triage algorithms includes: START (Simple Treatment and Rapid Transport), STM (Sacco Triage Method), Care Flight Triage and the Triage Sieve. ${ }^{[2,8-11]}$ On the other hand secondary triage is done to establish the order in which patients receive care at the hospital or, in the setting of delayed transportation, at the scene. ${ }^{[8]}$ It is usually done at patient referral sites, usually hospital emergency centres using major incident 
secondary triage algorithms such as Secondary Assessment of Victim Endpoint (SAVE) Triage or Triage Sort. ${ }^{[2,12]}$. However, triage protocols are not $100 \%$ accurate and none of them is applicable to all hazards. Further, there is no universally accepted major incident triage protocol that is well validated and reliable. . ${ }^{[27]}$

In major incident management, triage accuracy is critically important and Prehospital personnel are expected to perform quick and accurate triage. Specific triage protocols allow objective assessment of patients according to pre-set algorithms in order to improve accuracy and reliability. ${ }^{[13]}$. Triage errors occur when patients are inappropriately classified. There are two types: over triage and undertriage. Undertriage occurs when victims with life threatening injuries requiring immediate treatment are inappropriately classified to receive delayed care. Over triage occurs where victims with non-critical injuries are classified as urgent and requiring immediate care. This results in inappropriate assignment of resources and can hinder the effective management of critically injured patients. ${ }^{[14,15]}$ In mass casualty situations, where demand is high and resources low, the early principles of triage are critical in the delivery of effective emergency care with minimum time to treatment. To achieve this, triage systems must evolve to send the most appropriate responder to a particular situation. ${ }^{[3]}$ In a retrospective study of the Turkish Airline crash, the researchers emphasized the critical importance of triage accuracy in major incident management. Of the 135 victims, triaged by ambulance teams, an over triage rate up to $89 \%$ and an undertriage rate of $12 \%$ was found. ${ }^{[16,17]}$ While this had no impact on the mortality rate, due to the availability of resources in the region of the crash, it would have been worthwhile to evaluate the amount of resources that would have been saved with appropriate triage, and the cadre of medical personnel who performed the triage. In a Dutch study, the rate of undertriage was $10.9 \%$ (95\% CI 7.4-15.7) with the overall rate of overtriage being $39.5 \%(95 \%$ CI $36.9-42.1) .{ }^{[18]}$

One reason for errors in the triage of patients in major incident is the lack of adherence to the triage algorithm. Fitzharris et al conducted a retrospective evaluation of adherence to the New 
South Wales (NSW) prehospital triage protocol for trauma used by the ambulance service. Variations in performance across the different levels of paramedics in the NSW ambulance system were found. Adherence rates $(77 \%)$ were highest among the lowest-level trained personnel. ${ }^{[19]}$ In a prospective study of the Dutch Field Triage protocol for mass casualty patients, the researchers assessed the compliance to protocol by EMS personnel in the triage of 1607 victims of high energy impact trauma. ${ }^{[18]}$ Compliance to the triage protocol was $78.7 \%$ as 310 patients were not transported to the required level one or level two trauma centre. Similar findings of protocol non-adherence were reported by Wong et al in their Hong Kong study. ${ }^{[20]}$ They assessed appropriate diversion of 141 major trauma cases by paramedics, and found that the over-diversion rate was $3.5 \%$ with under-diversion at $40.5 \%$; overall accuracy was $74.5 \% .^{[20]}$

\section{Motivation}

The Western Cape EMS provides 24-hour medical response and pre-hospital medical care routinely and also during major incidents. EMS ambulances are staffed by Basic Life Support (BLS), Intermediate Life Support (ILS) and Advanced Life Support (ALS) prehospital emergency care practitioners. ${ }^{[21]}$ They are, the first medical professionals providing care in any major incident, and is therefore one of the main target groups for major incident training.

One of the key activities for prehospital emergency care practitioners in a major incident is triage of patients. Accurate triage is essential to proper patient management, appropriate referral and utilisation of resources. By evaluating triage accuracy across the various levels of prehospital emergency care practitioners, the researchers anticipate to make recommendations for the most reliable workforce for major incident triage. This will not only be a better 
utilization of resources but will also reduce errors in triage, and early initiation of appropriate treatment and transport to referral sites.

\section{Research Question}

What is the difference in mass casualty triage performance between differently skilled prehospital emergency care practitioners?

\section{Aims and objectives}

The aim of the study is to determine the difference in mass casualty triage performance between differently skilled pre-hospital emergency care practitioners.

The objectives are:

i. To validate the written assessment tool to determine triage accuracy.

ii. To determine and compare accuracy of different prehospital providers when using the triage sieve protocol.

iii. To determine and compare the duration of triage between different prehospital providers when using the triage sieve protocol

\section{METHODOLOGY}

\section{Study design}

Cross sectional study

\section{Study setting}

Major incident management in the Western Cape involves coordinated efforts between different teams such as the Fire and Rescue Service, the South African Police Service, and Emergency Medical Services (EMS). EMS provides 24-hour medical response, routine pre- hospital medical care and medical care during major incidents. EMS resources include 542 
vehicles, 54 bases and 2366 personnel, providing specified service levels of 176 ambulances per hour. ${ }^{[22]}$

The Western Cape (and the rest of South Africa) adopted the Major Incident Medical Management and Support (MIMMS) principles for major incident management as part of the FIFA 2010 Soccer World cup legacy. ${ }^{[23]}$ The triage system utilised in MIMMS is that of sieve (primary triage) and sort (secondary triage). ${ }^{[2]}$ It measures various physiological parameters that identify the priorities for treatment. Patients are categorised into one of four groups: 1) Red (priority 1) - patients' whose life is in immediate danger and requires immediate treatment; 2) Yellow (priority 2) - patients not in immediate danger, but do require urgent surgical or medical intervention 2 - 4 hours; 3) Green (priority 3) - patients with minor injuries; and 4) Blue (no priority) - patients who are either dead or has extensive injuries and cannot be saved with the limited resources available.

Scheduled MIMMS training is performed throughout the Western Cape during the year targeting various groups involved in major incident management- including EMS prehospital emergency care practitioners.

\section{Study Population}

All prehospital emergency care practitioners (BLS, ILS, ALS levels) undergoing major incident training within the Western Cape from February 2016 to July 2016, will be eligible for inclusion Inclusion Criteria:

- Registered with the Health Professions Council of South Africa

- Currently practicing pre-hospital medicine in South Africa. 
Exclusion Criteria:

- Prehospital emergency care practitioners who are trainers or have experience as trainers in major incident courses pertaining to MIMMS.

\section{Sample Size determination}

The study will use convenience sampling of all trainees during the data collection period (February to August 2016). However, the researchers have calculated the minimum sample size in each group that will be required to give statistical significance (power analysis). In the unlikely event that this number is not reached during the data collection period, the researchers will continue data collection beyond the stipulated time, to achieve this number.

Prior studies indicated variable triage accuracy rates in prehospital providers $( \pm 60 \%$ to $80 \%){ }^{[20 \text {, }}$ ${ }^{24,25]}$ We decided to use $70 \%$ as the expected accuracy for ALS providers. Assuming we expect a $10 \%$ difference in triage accuracy between each level of training ( 3 groups), 50 per group should be enough to give a power $>90 \%$ (significance level $5 \%$ ).

\section{Data collection}

Participants will be given three tests: 1) a written pre-test consisting of 20 multiple-choice questions; 2) a written post-test consisting of 20 multiple-choice questions; and 3) a face-toface post-training evaluation.

\section{Survey Tool Validation}

The pre-test and post-test will each have 20 multiple choice questions consisting of structured case scenarios (vignettes) with patients of major incidents requiring triage. The multiple-choice questions will be validated before the start of the study. The investigators will create 20 different multiple-choice questions. These questions will be distributed to 3 physicians considered to be experts based on their knowledge, training and experience in pre-hospital medicine in South Africa, and specifically the use of the triage sieve algorithm for major incidents. They will each independently answer the 20 questions. Any differences in answers between experts will 
be discussed afterwards and the correct answer decided by consensus agreement between the experts. The investigators will facilitate the consensus process. The validated questions will then be used for data collection.

The face-to-face evaluation will be done with a trainer where additional pressure is placed on the participant. This will be done using Triage cards and Table Top exercises that are part of the standard MIMMS training.

\section{Procedure}

At the start of major incident training, all attendees will be informed about the study. Those willing to participate will sign an informed consent form (Appendix 1).

\section{Phase 1:}

Consented participants will complete a validated written pre-test (20 randomly selected multiple-choice questions) to assess their accuracy at triaging major incident patients using the triage sieve algorithm.

Phase 2:

All prehospital emergency care practitioners will undergo the formal major incident training inclusive of the Triage Sieve system. To reduce bias, course trainers will be consistent throughout the data collection period. Practitioners not participating in the study will receive the same standard of teaching.

Phase 3:

After training completion, participants will undergo two post-tests; a written test similar to the pre-test (i.e. 20 multiple-choice questions) and a face-to-face evaluation with a trainer using triage cards and table-top scenarios where additional pressure is placed on participants. 


\section{Measures}

Variable to be collected are:

- Demographics (gender, age)

- $\quad$ Level of training (BLS, ILS, ALS levels)

- Year when relative level of training completed

- Previous MIMMS training

- Time taken to complete pre-test, post-test and face-to-face scenarios

Two outcomes will further be measured to assess participants' mass casualty triage performance:

1. Triage accuracy

2. Duration of triage

Triage accuracy will be measured as accuracy comparing the 'assigned' with the 'expected' triage category. The 'assigned' triage category will be defined as the triage category actually assigned by the participants. The 'expected' triage category will be considered the triage given by the expert group. The results will be presented as percentage of 'correct' triage, overtriage and undertriage.

The duration of triage will be considered the total time for completing triage during the faceto-face evaluation.

Data cleaning and entry into an SPSS worksheet will be done after every training course. Thereafter all test score sheets will be safely stored to enable future access. At the end of the study period, data will be cross checked for completeness before analysis. 


\section{Data analysis}

Data analysis will be done by the principal investigator with support from the supervisors using Epi DATA and analysed using SPSS 17.

Summary statistics will be used to describe all variables. Categorical data will be summarised using frequency counts or percentages, and distributions of variables will be presented as twoway tables or bar charts. Medians or means will be used as the measures of central tendency for continuous responses and standard deviations or quartiles as indicators of spread.

The relationships between continuous response variables and nominal input variables will be analysed using appropriate analysis of variance (ANOVA). When ordinal response variables are compared versus a nominal input variable, non-parametric ANOVA methods will be used: for completely randomized designs the Mann-Whitney test or the Kruskal-Wallis test will be used and for repeated measures the Wilcoxon- or Friedman tests will be used.

The relation between two nominal variables will be investigated with contingency tables and likelihood ratio chi-square tests. A p-value of $p<0.05$ will represent statistical significance in hypothesis testing and $95 \%$ confidence intervals will be used to describe the estimation of unknown parameters.

A STROBE checklist for cross-sectional studies will guide the final report writing. ${ }^{[26]}$

\section{Ethical and legal considerations}

Permission for the study will be obtained from the Western Cape EMS Management after receiving ethical clearance.

The study involves medical professionals who use English as their official language of communication, therefore there is no need to translate the consent form into either Afrikaans or Xhosa. 
Autonomy: Participation in the study is voluntary. Signed informed consent (Appendix 1) will be obtained from all participants and they will be free to withdraw their participation in the study at any time without any penalisation.

Justice: No personal identification from the participants is required and confidentiality of participants will be guarded. Participants will not be penalised if they decide not to participate or withdraw from the study. The collected data will be entered in a password protected electronic spreadsheet only accessible by the research team.

Beneficence: Participants will not benefit directly from the study, although feedback will be given as part of the MIMMS training. We foresee that the results will benefit the general community in future as we'll be able to determine the most appropriate level of responders to be dispatched when major incidents occur, thereby potentially improving the quality and therefore outcomes of medical management provided.

Non-maleficence: There is minimal risk to the participants included in the study.

\section{Strengths and limitations}

Strengths: This is a prospective study, which makes the data collection process more complete as data can be checked for completeness prior to entering in system.

The study is designed to coincide with scheduled Western Cape Provincial major incident trainings for pre-hospital providers, making it easier to access participants.

Limitations: The study will not include volunteers and other non-HPCSA registered prehospital providers. Even though in the event of mass casualty incidents they are often part of the prehospital response team, they are not a constant part of the health system and therefore, further training and evaluation will not be possible. 


\section{Data dissemination plan}

While this study is being done as partial fulfilment for a Masters in Medicine degree, the investigators expect to publish an original article or short report in a peer reviewed journal. Results will also be presented at a national or international Emergency Medicine conference.

The study findings will also be shared with the Western Cape EMS Management. 


\section{Project timeline}

\begin{tabular}{|l|c|c|c|c|c|c|c|c|c|c|c|c|}
\hline 2015 & JAN & FEB & MAR & APR & MAY & JUN & JUL & AUG & SEP & OCT & NOV & DEC \\
\hline SUN HREC APPROVAL & & & & & & & & & & $\mathbf{X}$ & $\mathbf{X}$ & $\mathbf{X}$ \\
\hline 2016 & & & & & & & & & & & \\
\hline EMS APPROVAL & $\mathbf{X}$ & & & & & & & & & & & \\
\hline $\begin{array}{l}\text { PRE-TEST AND POST- } \\
\text { TEST VALIDATION }\end{array}$ & $\mathbf{X}$ & $\mathbf{X}$ & & & & & & & & & & \\
\hline DATA COLLECTION & & $\mathbf{X}$ & $\mathbf{X}$ & $\mathbf{X}$ & $\mathbf{X}$ & $\mathbf{X}$ & $\mathbf{X}$ & & & & & \\
\hline DATA MANAGEMENT & & & $\mathbf{X}$ & $\mathbf{X}$ & $\mathbf{X}$ & $\mathbf{X}$ & $\mathbf{X}$ & $\mathbf{X}$ & & & & \\
\hline DATA ANALYSIS & & & & & & & & & $\mathbf{X}$ & & & \\
\hline $\begin{array}{l}\text { COMPILATION OF } \\
\text { FINAL REPORT }\end{array}$ & & & & & & & & & & $\mathbf{X}$ & $\mathbf{X}$ & \\
\hline SUBMISSION & & & & & & & & & & & & $\mathbf{X}$ \\
\hline
\end{tabular}

\section{Resources and budget}

The study is self-funded. However, alternative funding will be sought from Harry Crossley Foundation.

Data Collection will occur during the scheduled training courses. Therefore there will be no costs incurred for training the study participants. However, adequate budgetary collection will go towards coordination, travel to site and data collection (printing and photocopying pre and post-tests). Data entry and analysis will be done by the researchers. 


\begin{tabular}{|l|l|l|l|l|}
\hline ITEM & DESCRIPTION & $\begin{array}{l}\text { UNIT } \\
\text { COST }\end{array}$ & $\begin{array}{l}\text { NUMBER OF } \\
\text { UNITS }\end{array}$ & $\begin{array}{l}\text { TOTAL } \\
\text { COST }\end{array}$ \\
\hline CONSUMABLES & & & & 5000 \\
\hline $\begin{array}{l}\text { Printing \& reproduction } \\
\text { (data collection) }\end{array}$ & Consent forms & 5 & 1000 & 5000 \\
\hline & Pre-test & 5 & 1000 & 5000 \\
\hline $\begin{array}{l}\text { Printing \& reproduction } \\
\text { (thesis) }\end{array}$ & Fost-Test & 5 & 1000 & 200 \\
\hline RESEARCH TRAVEL & 12 Sessions & & - & 20 \\
\hline Travel to site* & & 10 & & 3000 \\
\hline PERSONEL Thesis & 0 & 0 & 0 & 0 \\
\hline Statistician & 0 & 0 & 0 & 18,200 \\
\hline Research Assistant & & & & \\
\hline TOTAL & & & & \\
\hline
\end{tabular}

*The training schedule for 2016 still needs to be finalised. The R3000 is therefore just an estimate. The calculation is based on $\mathrm{R} 3.50 / \mathrm{km}$ and an average of $70 \mathrm{~km}$ per training session. 


\section{References}

1. Mackway-Jones K. Major Incident Medical Management and Support: The Practical Approach at the Scene: John Wiley \& Sons; 2012.

2. Mackway-Jones K. Triage. Major Incident Medical Management and Support: The Practical Approach at the Scene: Wiley-Blackwell; 2011. p. 89-102.

3. Robertson-Steel I. Evolution of triage systems. Emerg Med J. 2006 Feb;23(2):154-5.

4. Baker MS. Creating order from chaos: part I: triage, initial care, and tactical considerations in mass casualty and disaster response. Mil Med. 2007 Mar;172(3):232-6.

5. Lax P, Prior K. Major incident pre-hospital care. Surgery (Oxford). 2015 9//;33(9):41923.

6. Vassallo J, Horne S, Ball S, Whitley J. UK Triage the validation of a new tool to counter an evolving threat. Injury. $2014 \mathrm{Dec} ; 45(12): 2071-5$.

7. Bostick NA, Subbarao I, Burkle FM, Jr., Hsu EB, Armstrong JH, James JJ. Disaster triage systems for large-scale catastrophic events. Disaster Med Public Health Prep. 2008 Sep;2 Suppl 1:S35-9.

8. Jenkins JL, McCarthy ML, Sauer LM, et al. Mass-casualty triage: time for an evidencebased approach. Prehosp Disaster Med. 2008 Jan-Feb;23(1):3-8.

9. $\quad$ START background. Critical Illness and Trauma Foundation, Inc.; [cited 2015 September]; Available from: http://citmt.org/Start/background.htm.

10. Sacco WJ, Navin DM, Fiedler KE, Waddell RK, 2nd, Long WB, Buckman RF, Jr. Precise formulation and evidence-based application of resource-constrained triage. Acad Emerg Med. 2005 Aug;12(8):759-70.

11. Garner A, Lee A, Harrison K, Schultz CH. Comparative analysis of multiple-casualty incident triage algorithms. Annals of emergency medicine. 2001 Nov;38(5):541-8.

12. Benson M, Koenig KL, Schultz CH. Disaster triage: START, then SAVE--a new method of dynamic triage for victims of a catastrophic earthquake. Prehosp Disaster Med. 1996 Apr-Jun;11(2):117-24.

13. Martí R, Robles S, Martín-Campillo A, Cucurull J. Providing early resource allocation during emergencies: The mobile triage tag. Journal of Network and Computer Applications. 2009;32(6):1167-82.

14. Frykberg E. Triage: principles and practice. Scandinavian journal of surgery. 2005;94(4):272.

15. Frykberg ER. Medical management of disasters and mass casualties from terrorist bombings: how can we cope? J Trauma. 2002 Aug;53(2):201-12.

16. Postma IL, Weel H, Heetveld MJ, et al. Patient distribution in a mass casualty event of an airplane crash. Injury. 2013 Nov;44(11):1574-8.

17. Postma IL, Weel H, Heetveld MJ, et al. Mass casualty triage after an airplane crash near Amsterdam. Injury. 2013 Aug;44(8):1061-7.

18. van Laarhoven JJ, Lansink KW, van Heijl M, Lichtveld RA, Leenen LP. Accuracy of the field triage protocol in selecting severely injured patients after high energy trauma. Injury. 2014 May;45(5):869-73. 
19. Fitzharris M, Stevenson M, Middleton P, Sinclair G. Adherence with the pre-hospital triage protocol in the transport of injured patients in an urban setting. Injury. 2012 Sep;43(9):1368-76.

20. Wong C, Lui C, So F, Tsui K, Tang S. Prevalence and predictors of under-diversion in the primary trauma diversion system in Hong Kong. Hong Kong Journal of Emergency Medicine. 2013;20(5):276.

21. Western Cape Government. Medical Emergency Transport and Rescue. [cited 2015 September]; Available from: https://www.westerncape.gov.za/service/medical-emergencytransport-and-rescue-metro.

22. City of Cape Town. City of Cape Town Draft Annual Report 2012/13 [cited 201521 September]: Available from: http://mfma.treasury.gov.za/Documents/06.\%20Annual\%20Reports/201213/01.\%20Metros/CPT\%20Cape\%20Town/CPT\%20Cape\%20Town\%20Draft\%20Annual\%2 0Report\%202012_13.pdf.

23. Smith W. Triage in mass casualty situations. Continuing Medical Education. 2012;30(11):413-5.

24. Kilner T. Triage decisions of prehospital emergency health care providers, using a multiple casualty scenario paper exercise. Emerg Med J. 2002 Jul;19(4):348-53.

25. Deluhery MR, Lerner EB, Pirrallo RG, Schwartz RB. Paramedic accuracy using SALT triage after a brief initial training. Prehosp Emerg Care. 2011 Oct-Dec;15(4):526-32.

26. Knottnerus A, Tugwell P. STROBE--a checklist to Strengthen the Reporting of Observational Studies in Epidemiology. J Clin Epidemiol. 2008 Apr;61(4):323.

27. Jenkins JL, McCarthy ML, Sauer LM, Green GB, Stuart S, Thomas TL, Hsu EB: Masscasualty triage: time for an evidence-based approach. Prehospital Disast Med 2008; $23: 3-8$ 


\section{Appendices}

Appendix 1 - Consent form

\section{PARTICIPANT INFORMATION LEAFLET}

A COMPARISON BETWEEN DIFFERENTLY SKILLED PREHOSPITAL EMERGENCY

CARE PRACTITIONERS IN MASS CASUALTY TRIAGE IN SOUTH AFRICA

REFERENCE NUMBER:

PRINCIPAL INVESTIGATOR: DR. ANNET ALENYO NGABIRANO

ADDRESS: Division of Emergency Medicine at Stellenbosch University

\section{CONTACT NUMBER:Copyright}

Dear Colleague,

My name is Dr. Annet Alenyo Ngabirano and I am an Emergency Medicine Registrar from the division of Emergency Medicine at Stellenbosch University. I would like to invite you to participate in a research project that aims to compare differently skilled prehospital emergency care practitioners in mass casualty triage in South Africa. 
Please take some time to read the information presented here, which will explain the details of this project and contact me if you require further explanation or clarification of any aspect of the study. Also, your participation is entirely voluntary and you are free to decline to participate. If you say no, this will not affect you negatively in any way whatsoever. You are also free to withdraw from the study at any point, even if you do agree to take part.

This study has been approved by the Health Research Ethics Committee (HREC) at Stellenbosch University and will be conducted according to accepted and applicable National and International ethical guidelines and principles, including those of the international Declaration of Helsinki October 2008.

The study is being done among prehospital emergency care practitioners undergoing major incident training between February 2016 and July 2016. You have been identified as a potential participant in this study because you are a prehospital emergency care practitioner (Basic, Intermediate OR Advanced level) registered with the HPCSA and currently practicing prehospital medicine in South Africa.

\section{PURPOSE OF THE STUDY}

To evaluate triage accuracy across the three various levels of prehospital emergency care practitioners and make recommendations for the most reliable workforce for major incident triage. This will not only be a better utilization of resources but will also reduce errors in triage, and early initiation of appropriate treatment and transport to referral sites. If you agree to participate in this study then the following will happen:

- You will be asked to avail information about your age, gender, training and duration of service in prehospital care.

- You will write a pre-test before the MIMMS training course

- You will then undergo the training

- You will then complete two post-tests after the course: a classroom setting test and a face-to-face table top exercise with the trainers. 


\section{BENEFITS:}

The study will not directly benefit study participants. However, through recommendations from the study, the general community will benefit from improved major incident preparedness and response activities. By recommending appropriate workforce for triage, resources can be diverted for other purposes.

\section{RISKS:}

There is minimal risk to study participants.

Your identity will not be used in any reports or publications resulting from this study, all records will be kept confidential and no unauthorized person shall have access to it.

\section{COSTS:}

You will not be paid to participate in this study, and you will also not incur any costs to participate.

\section{RIGHT TO REFUSE OR WITHDRAW:}

Your participation in this study is entirely voluntary and no consequences will result if you refuse to participate. If you either refuse or withdraw from the study, you will still continue with your MIMMS training as per the normal standard.

\section{QUESTIONS:}

In case of questions or problems related to the study, you can ask now or contact: Dr. Annet Alenyo Ngabirano, Emergency Medicine Registrar, Division of Emergency Medicine at Stellenbosch University, on Copyright

If you have any concerns or complaints that have not been adequately addressed by the study team, you can contact the Stellenbosch University Health Research Ethics Committee at Cop 
If you are willing to participate in this study please sign the attached Declaration of Consent and hand it to the investigator.

Yours sincerely

Dr. Annet Alenyo Ngabirano

Principal Investigator 


\section{Appendix 2: Declaration by participant}

By signing below, agree to take part in the research study entitled A COMPARISON BETWEEN DIFFERENTLY SKILLED PREHOSPITAL EMERGENCY CARE PRACTITIONERS IN MASS CASUALTY TRIAGE IN SOUTH AFRICA.

I declare that:

- I have read the attached information leaflet and it is written in a language with which I am fluent and comfortable.

- I have had a chance to ask questions and all my questions have been adequately answered.

- I understand that taking part in this study is voluntary and I have not been pressurised to take part.

- I may choose to leave the study at any time and will not be penalised or prejudiced in any way.

- I may be asked to leave the study before it has finished, if the researcher feels it is in my best interests, or if I do not follow the study plan, as agreed to.

Signed at (place) On (date) 2016

Signature of participant: 


\section{Appendix 3: Survey tool}

SURVEY TOOL:

A COMPARISON BETWEEN DIFFERENTLY SKILLED PREHOSPITAL EMERGENCY

CARE PRACTITIONERS IN MASS CASUALTY TRIAGE IN SOUTH AFRICA

Study number:

Training Site:

Date:

SECTION A: DEMOGRAPHICS:：(TO BE COMPLETED BY PARTICIPANTS)

\begin{tabular}{|c|c|c|c|c|c|c|}
\hline $\begin{array}{l}\text { Age } \\
\text { (Years) }\end{array}$ & & & & & & \\
\hline $\begin{array}{l}\text { Sex: } \\
\text { (tick) }\end{array}$ & MALE & $\mathrm{FH}$ & IALE & OTHER & (IFY): & \\
\hline $\begin{array}{l}\text { Duration } \\
\text { of Service } \\
\text { (in years): }\end{array}$ & $<1$ Year & 1-2 Years & 2-3 Years & 3-4 Years & Years & $>5$ Years \\
\hline & & & & & & \\
\hline
\end{tabular}


SECTION B: TRAINING: (TO BE COMPLETED BY INVESTIGATORS)

\begin{tabular}{|l|l|l|l|}
\hline & BLS & ILS & ALS \\
\hline Level of Training (tick): & & & \\
\hline Year Completed & & & \\
& & & \\
\hline
\end{tabular}

Phase 1: Pre-test

\begin{tabular}{|l|l|l|}
\hline Correct Triage & Over Triage & Undertriage \\
\hline & & \\
\hline & & \\
\hline
\end{tabular}

Phase 2:

\begin{tabular}{|l|l|l|}
\hline & Yes & No \\
\hline MIMMS training completed: & & \\
\hline
\end{tabular}

Phase 3:

Written Post-test:

\begin{tabular}{|l|l|l|}
\hline Correct Triage & Over Triage & Undertriage \\
\hline & & \\
\hline & & \\
\hline
\end{tabular}

Face-to-face:

\begin{tabular}{|l|l|l|l|}
\hline Correct Triage & Over Triage & Undertriage & Time taken to complete triage \\
\hline & & & \\
\hline
\end{tabular}




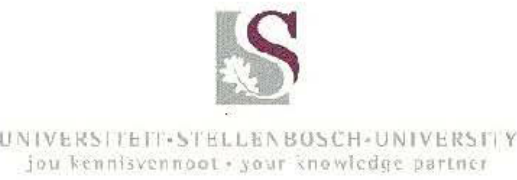

\section{Approved with Stipulations}

New Application

27-Nov-2015

Alenyo Ngabirano, Annet AAN

Ethics Reference \#: S15/10/238 Title: $\quad$ A comparison between differently skilled prehospital emergency care practitioners in mass casualty triage in South
Africa.

Dear Dr Annet Alenyo Ngabirano,

The New Application received on 26-Oct-2015, was reviewed by Health Research Ethics Committee 2 via Committee Review procedures on 18-Nov-2015.

Please note the following information about your approved research protocol

Frotocol Approval Period: 18-Now-2015 -18-Nov-2016

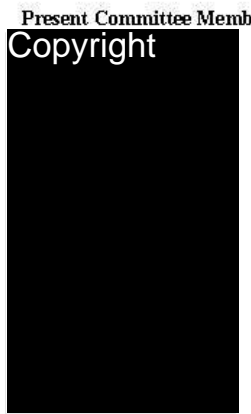

The Stipulations of your ethics approwal are as follows:

1. Please refer to the 2013 Declaration of Helsinki not 2008.

2. Also state that ethics approval was sought from the Stellenbosch University HREC.

Please remember to use your protocol number (S15/10/238) on any documents or correspondence with the HREC concerning your research protocol.

Please note that the $\mathrm{HREC}$ has the prerogative and authority to ask further questions, seek additional information, require further modifications, or monitor the conduct of your research and the consent process.

\section{After Ethical Review:}

Please note a template of the progress report is obtainable on www.sun.acza/rds and should be submitted to the Committee before the year has expired. The Committee will then consider the continuation of the project for a further year (if necessary). Annually a number of projects may be selected randomly for an external audit.

Translation of the consent document to the language applicable to the study participants should be submitted.

Federal Wide Assurance Number: 00001372

Institutional Review B oard (IRB) Number: IRB 0005239 
The Health Research Ethics Committee complies with the SA National Health Act No.61 2003 as it pertains to health research and the United States Code of Federal Regulations Title 45 Part 46. This committee abides by the ethical norms and principles for research, established by the Declaration of Helsinki, the South African Medical Research Council Guidelines as well as the Guidelines for Ethical Research: Principles Structures and Processes 2004 (Department of Health).

\section{Provincial and City of Cape Town Approval}

Please note that for research at a primary or secondary healthcare facility permission must still be obtained from the relevant authorities (Western Cape Department of Health and/or City Health) to conduct the research as stated in the protocol. Contact persons are Copyright at Western Cape Department of Health Copyright Tel: Convrioh) and Copyright at City HealthConvriaht

Copyright . Research that will be conducted at any tertiary academic institution requires approval from the relevant hospital manager. Ethics approval is required BEFORE approval can be obtained from these health authorities.

We wish you the best as you conduct your research.

For standard HREC forms and documents please visit: www.sun.ac.za/rds

If you have any questions or need further assistance, please contact the HREC office at CODVri

Included Documents:

PICF

Protocol

CV N van Hoving

Declaration A Ngabirano

Checklist

Declaration $\mathrm{N}$ van Hoving

Application form

CV A Ngabirano

Protocol Synopsis

Sincerely,

\section{Copyright}

HREC Coordinat or

Health Research Ethics Committee 2 


\section{Investigator Responsibilities}

\section{Protection of Human Research Participants}

\section{Some of the responsibilities investigators have when conducting research involving human participants are listed below:}

1.Conducting the Research. You are responsible for making sure that the research is conducted according to the HREC approved research protocol. You are also responsible for the actions of all your co-investigators and research staff involved with this research.

2.Participant Enrolment. You may not recruit or enrol participants prior to the HREC approval date or after the expiration date of HREC approval. All recruitment materials for any form of media must be approved by the HREC prior to their use. If you need to recruit more participants than was noted in your HREC approval letter, you must submit an amendment requesting an increase in the number of participants.

3.Informed Consent. You are responsible for obtaining and documenting effective informed consent using only the HREC-approved consent documents, and for ensuring that no human participants are involved in research prior to obtaining their informed consent. Please give all participants copies of the signed informed consent documents. Keep the originals in your secured research files for at least fifteen (15) years.

4.Continuing Review. The HREC must review and approve all HREC-approved research protocols at intervals appropriate to the degree of risk but not less than once per year. There is no grace period. Prior to the date on which the HREC approval of the research expires, it is your responsibility to submit the continuing review report in a timely fas hion to ensure a lapse in HREC approval does not occur. If HREC approval of your research lapses, you must stop new participant enrolment, and contact the HREC office immediately.

5.Amendments and Changes. If you wish to amend or change any aspect of your research (such as research design, interventions or procedures, number of participants, participant population, informed consent document, instruments, surveys or recruiting material), you must submit the amendment to the HREC for review using the current Amendment Form. You may not initiate any amendments or changes to your research without first obtaining written HREC review and approval. The only exception is when it is necessary to eliminate apparent immediate hazards to participants and the HREC should be immediately informed of this necessity.

6. Adverse or Unanticipated Events. Any serious adverse events, participant complaints, and all unanticipated problems that involve risks to participant or others, as well as any research-related injuries, occurring at this institution or at other performance sites must be reported to the HREC within five (5) days of discovery of the incident. You must also report any instances of serious or continuing problems, or non-compliance with the HRECs requirements for protecting human research participants. The only exception to this policy is that the death of a research participant must be reported in accordance with the Stellenbosch Universtiy Health Research Ethics Committee Standard Operating Procedures www.sun025.sun.ac.za/poital /page/portal/Health Sciences/English/Centres\%20and\%20Institutions/Research Development Support/Ethics/Application package All reportable events should be submitted to the HREC using the Serious Adverse Event Report Form.

7.Research Record Keeping. You must keep the following research-related records, at a minimum, in a secure location for a minimum of fifteen years: the HREC approved research protocol and all amendments; all informed consent documents; recruiting materials; continuing review reports; adverse or unanticipated events; and all correspondence from the HREC

8.Reports to the MCC and Sponsor. When you submit the required annual report to the MCC or you submit required reports to your sponsor, you must provide a copy of that report to the HREC. You may subnit the report at the time of continuing HREC review.

9.Provision of Emergency Medical Care. When a physician provides emergency medical care to a participant without prior HREC review and approval to the extent permitted by law, such activities will not be recognised as research nor will the data obtained by any such activities should it be used in support of research

10.Final reports. When you have completed (no further participant enrolment, interactions, interventions or data analysis) or stopped work on your research, you must submit a Final Report to the HREC.

11.On-Site Evaluations, MCC Inspections, or Audits. If you are notified that your research will be reviewed or audited by the MCC, the sponsor, any other external agency or any internal group, you must inform the HREC immediately of the impending audit/evaluation 


\section{S}

UNIVERSTTEIT-STELLENB OSCH.UNIVERSITY

jou kennisvennool. your knowledge fartier

03-Nov-2016

\section{Ethics Letter}

Alenyo Ngabirano, Annet AAN

Ethics Reference \#: S15/10/238

Title: A comparison between differently skilled prehospital emergency care practitioners in mass casualty triage in South A frica.

Dear Dr Annet Alenyo Ngabirano

Your request for extension/annual renewal of ethics approval dated 28 October 2016 refers.

The Health Research Ethics Committee reviewed and approved the annual progress report you submitted through an expedited process.

The approval of the research project is extended for a further year.

Approval date: 03 November 2016

Expiry date: 02 November 2017

Where to sub mit any doc umentation

Kindly submit ONE HARD COPY to Elvira Rohland, RDSD, Room 5007, Teaching Building, and ONE ELECTRON IC COPY to ethics@sun.ac.za

Please remember to use yourp rotocol number (S15/10/238) on any documents or correspondence with the HREC concerning your research protocol.

Federal Wide Assurance Number. 00001372

Institutional Review B oard (IRB) Number. IRB 0005240 for HREC1

Institutional Review B oard (IRB) Number. IRB 0005239 for HREC2

The Health Research Ethics Committee complies with the SA National Health A ct No.61 2003 as it pertains to health research and the United States Code of Federal Regulations Title 45 Part 46. This committee abides by the ethical norms and principles for research, established by the Declaration of Hel sinki, the South A frican Good Clinical Pracices Guidelines as well as the Guidelines for Ethical Research: Principles Structures and Processes 2004 (Department of Health).

\section{Sincerely,}

\section{Copyright}

\section{REC Coordinato}

Health Research Ethics Committee 2 


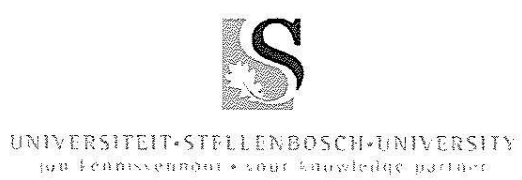

Ethics Letter

07-Mar-2018

HREC Reference \#: S15/10/238

Title: A comparison between differently skilled prehospital emergency care practitioners in mass casaulty triage in South Africa

Dear Dr Annet Alenyo-Ngabirano,

Your request for extension/annual renewal of ethics approval dated 17 November 2017 refers.

The Health Research Ethics Committee reviewed and approved the annual progress report you submitted through an expedited review process.

The approval of the research project is extended for a further year.

\section{Approval Date: 07 March 2018}

Expiry Date: 06 March 2019

Kindly be reminded to submit progress reports two (2) months before expiry date.

\section{Where to submit any documentation}

Kindly submit ONE HARD COPY to Elvira Rohland, RDSD, Room 5007, Teaching Building, and ONE ELECTRONIC COPY to ethics@sun.ac.za.

Please remember to use your HREC reference number (S15/10/238) on any documents or correspondence with the HREC concerning your research protocol.

National Health Research Ethics Council (NHREC) Registration Numbers: REC-130408-012 for HREC1 and REC-230208-010 for HREC2

Federal Wide Assurance Number: 00001372

Institutional Review Board (IRB) Number: IRB0005240 for HREC1

Institutional Review Board (IRB) Number: IRB0005239 for HREC2

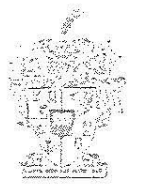

Fatulate Geneskunde en Gesondheidswetershape

Faculty of Medicine and Health Sciences

Afdeling Navorsingsontwikkeling en -Steun OResearch Development and Support Division

Posbus/PO Box 241 - Cape Town 8000 - Suid-Afrika/Souch Africa

Tel: $+27(0) 219389677$ 


\section{$\mathrm{S}$ \\ UNMTRSITET-5TELLNBOSCH.UNWERSIT

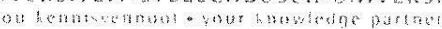

The Health Research Ethics Committee complies with the SA National Health Act No. 61 of 2003 as it pertains to health research and the United States Code of Federal Regulations Title 45 Part 46 . This committee abides by the ethical norms and principles for research, established by the Declaration of Helsinki and the South African

Medical Research Council Guidelines as well as the Guidelines for Ethical Research: Principles, Structures and Processes 2015 (Departement of Health).

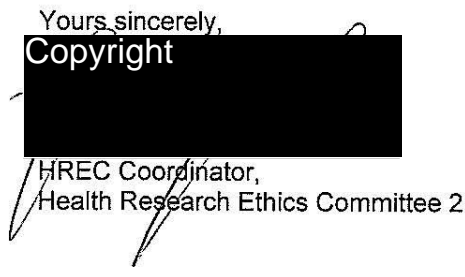

STELLENBOSCH UNIVERSITY

Health Research Ethics Committee

\section{MAR 2018}

STELLENBOSCH UNIVERSITEIT

Gesondheidsnavorsing Etiekkomittee

Fahulteit Geneeskunde en Gesondheidswetenshappe

Faculy of Medicine and Health Sciences

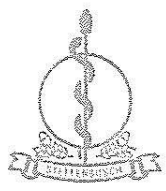
s.

Aideling Nayorsingsontwikkeling en -Steun Research Development and Support Division

Posbus/PO Box 24I • Cape Town 8000 - Suid-Afrika/South Africa

Tel: +27 (0) 219389677 


\section{3) APPROVAL FROM WESTERN CAPE DEPARTMENT OF HEALTH}

DIRECTORATE: EMERGENCY MEDICAL SERVICES

ENQUIRIES: Copyright

Government

\section{Attention: Dr Annet Alenyo}

\section{RE: REQUEST FOR PERMISSION TO CONDUCT RESEARCH - ' A COMPARISON BETWEEN DIFFERENTLY SKILLED EMERGENCY CARE PRACTITIONERS IN MASS CASUALTY TRIAGE IN SOUTH AFRICA'}

Dear Dr Annet Alenyo,

Your request on the above matter refers.

Thank you for the request to conduct research within the Western Cape Government Emergency Medical Services. Your proposal has been evaluated by the Emergency Medicine Division Research Committee and has been recommended for approval by this office.

I am therefore pleased to inform you that such approval is hereby granted.

I wish you well in your endeavor and trust that you will keep this office and its department informed of your findings when these become available.

Yours sincerely

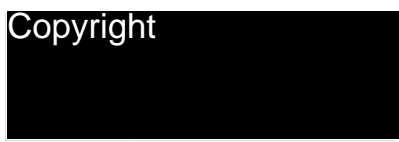

\section{Copyright}

\section{Copyriaht}

Western Cape Government Health

Date: $17^{\text {TH }}$ February 2016 


\section{4) INSTRUCTIONS FOR CONTRIBUTORS: Prehospital and Disaster Medicine}

Available from:

https://www.cambridge.org/core/journals/prehospital-and-disaster-

medicine/information/instructions-contributors

\section{Editorial Policy}

Manuscripts in one of six different categories can be submitted for review and publication in Prehospital and Disaster Medicine (PDM): (1) Original Research; (2) Brief Report; (3) Special Report; (4) Case Report; (5) Comprehensive Review; or (6) Field Report. Other types of manuscripts must have the approval of the Editor before being submitted. The characteristics specific to each of these categories are described below:

1. Original Research-structured research that uses quantitative and/or qualitative data collection methods and analyses to establish a hypothesis or prove a cause and effect relationship is included in this category. The manuscript must be structured as follows:

Abstract - concise summary (not to exceed 375 words) in the following format:

Introduction: Describes the need for the study

Hypothesis/Problem: What was tested (experimental studies only) If qualitative, statement of problem addressed.

Methods: Summary of methods used with subsections as appropriate (type of subjects, number of subjects, study design, statistical tests). If the work is qualitative, the types of instruments used and the scope and type of work must be described. Results: Summary of principal findings.

Conclusion: Implications of findings.

Introduction-provide justification for the effort with appropriate references annotated. If 
quantitative, the concluding sentence should define the hypothesis. If qualitative, the problem being addressed should be stated clearly.

Methods - detailed description of methods used, including type and number of subjects, study design, statistical tests, software and equipment. This section should be sufficiently detailed that other investigators would be able to reproduce the study. Statistical methods used must be annotated. Approval by an Institutional Review Committee must be included when appropriate.

Results - results must be presented in text using both numbers and percentages where applicable, and may be accompanied by tables and figures (tables and figures must be referred to in text). The text must explain all data included in tables and figures, but should not be unnecessarily redundant. All direct results from the study must appear in this section. No discussion of the results may be included.

Discussion - the discussion should provide an interpretation of the results in terms of meaning and application. Results should not be repeated. Computations or extrapolations that may help explain the results may be provided. Limitations of the study should be defined and suggestions for future research should be included. Also include references that support or negate explanations provided.

Conclusion - the findings in terms of implications for the practice of prehospital, emergency, and/or disaster (humanitarian) medicine should be summarized in a few sentences. No new information should be provided.

References - a numbered list of references in the order in which they appear in the text. References should not be formatted as footnotes.

2. Brief Report - a short report on work that does not meet all of the criteria required for original research. Preliminary reports also are included in this category. The format used should be identical to that used for Original Research.

3. Special Report - describes activities or aspects of our science that provide information 
necessary for the progression of the science. The manuscript should be structured as follows:

Abstract - concise summary (not to exceed 375 words)

Introduction - highlight the problem being addressed and the reasons that it needs to be addressed.

Report - describes an aspect of science or information. Any subsections should be subtitled. Include citations for sources of the material.

Discussion-describes the significance of the report in terms of the science. Includes a comprehensive review of the pertinent literature.

Conclusion - the findings in terms of implications for the practice of prehospital, emergency, and/or disaster (humanitarian) medicine should be summarized in a few sentences.

References - a numbered list of references in the order in which they appear in the text. References should not be formatted as footnotes.

4. Case Report - uses one or more cases of specific patients or events/responses to highlight a current aspect of medical care or a phenomenon. Case reports also may have value in the development of definitive research projects by the same or other investigators. The format for a Case Report is the same as that for Special Report described above.

5. Comprehensive Review - a review of the literature to be used to clarify areas in which there seems to be disagreement. Comprehensive reviews seek to establish the evidence base for the area being addressed. The format used should be identical to that described for Special Reports.

6. Field Report — analyze and provide direct observations of prehospital and disaster events. The manuscript should be structured as follows:

\section{Specific event identifiers, including:}

a. Event type (example: tropical storm, bombing, train crash, mass-gathering event); 
b. Event onset date;

c. Location of event (geographic area from which report is being made);

d. Geographic coordinates in latitude, longitude, elevation;

e. Dates (or times) of observations reported; and

f. Response type (example: medical relief, humanitarian, public health surveillance).

Abstract — limited to approximately 100 words (for indexing with literature search databases).

Introduction - summary of event with specific data available, such as population density; detailed event description; and general damage that occurred. Include description of author response responsibilities and mission objectives.

Source(s) - for information and data used for the report.

\section{Observations}

Analysis - of Observations with any recommendations.

References - a numbered list of references (if any used in analysis of observations) in the order in which they appear in the text. References should not be formatted as footnotes.

The main text of a field report submitted to Prehospital and Disaster Medicine should be no more than 1,500 words in length. Supporting maps, graphs, and tables are encouraged.

\section{Manuscript Submission}

All manuscripts must be submitted through the Journal's online submission platform, ScholarOne Manuscripts, at http://mc.manuscriptcentral.com/pdm. The following must be included with the submitted manuscript:

Cover Letter - each manuscript should be accompanied by a cover letter addressing the following: 
- The primary author attests to the original nature of the material, that the paper has not been published elsewhere, and is not under consideration by any other publication

- If the paper has been, or is to be presented in a forum orally or as a poster, indicate the title of the forum, sponsoring institution, and the date of presentation.

- The authors agree to transfer copyright to Prehospital and Disaster Medicine if accepted for publication

- The institution(s) in which the work was performed, the sponsoring institution(s), and the respective departments are annotated

- The name of the author to whom any correspondence should be directed, along with correspondence street address and email address

- Three to five keywords or phrases in alphabetical order separated by semicolons to facilitate indexing or electronic searches. Use the US National Library of Medicine Medical Subject Headings database (http://www.ncbi.nlm.nih.gov/mesh) to develop these keywords or phrases.

Cover Page - includes the title of the paper, first names, middle initials, last names, and highest academic degrees of all authors (abbreviated as MD, MPH, etc.), along with institutions each author is affiliated with. Reiterate from the cover letter the name of the author to whom any correspondence should be directed, and the street address and email address. Do not indicate author names or institutions anywhere in the manuscript other than on the cover page.

\section{Manuscript Format}

In general, manuscripts should be formatted using the AMA Manual of Style.

File Format—please submit manuscripts as Word or rich text files. DO NOT SUBMIT AS PDFs. 
Language - all manuscripts must be submitted in American English. Do not use $I$, We, or Our when describing the researchers. The fact that the research was conducted by the authors is implicit.

Type Style - use Times New Roman 12 point font. Double space all text, including references. Left-justify the text; leave right margins unjustified.

Abbreviations - provide a list of abbreviations used more than once and what they stand for at the beginning of the manuscript (example: WHO: World Health Organization). Whenever such abbreviations are used, they also must be annotated with the initial mention of words within the manuscript followed by the abbreviation in parentheses.

Generic Names - whenever possible, use generic names. Brand names may be indicated parenthetically and the name and location of the manufacturer must be provided in parentheses followed by a generic description of the medication, drug, product, or equipment.

Software and Equipment Descriptions - specify version number, name, manufacturer or developer of all software and equipment used. Include the city, state or province and country in which the manufacturer or developer is

headquartered. Example: the data were entered into a Microsoft Excel spreadsheet Version 7.0.25 (Microsoft Corporation, Redmond, Washington, USA).

Numbers and Percentages - both numbers and percentages should be presented in the text, in the $\mathrm{n}(\%)$ format.

Mean and Standard Deviation - when presenting means and standard deviations in the text, the mean (SD) format should be used, rather than the \pm format.

REFERENCES - references must be cited in the References section at the end of the manuscript in the order in which they appear in the text. Do not use automatic numbering, and remove any formatting (such as that from EndNote) linking the reference to citations 
in the text. References should not be formatted as footnotes. All references must be cited by superscript Arabic numbers in the text, tables, and legends for illustrations. Citations in the text should be placed after punctuation such as periods or commas. Titles of journals referenced must be annotated using US National Library of Medicine abbreviations (http://www.ncbi.nlm.nih.gov/nlmcatalog) and must be italicized. If there is no US National Library of Medicine abbreviation, please do not abbreviate the journal title. Include volume and issue numbers when possible, and do not omit digits from inclusive page numbers. The following format for references must be used:

Journals-White SJ, Hamilton WA, Veronesi JF. Comparison of field techniques used to pressure infuse intravenous fluids. Prehosp Disaster Med. 1991;6(4):429-434.

Books - Schwartz GR, Safar P, Stone JH, et al, eds. Principles and Practice of Emergency Medicine. 2nd ed. Philadelphia, PA, USA: WB Saunders Co.; 1985:1198-1202.

Chapters-Lindberg R. Pathology of head injuries. In: Cowley RA, Trump BF, eds, Pathophysiology of Shock. Baltimore, MD, USA: Williams and Wilkins; 1982:588-592.

Presentations at meetings (published)-Jones M. Lessons learned during the Haiti earthquake. Paper presented at: 17th Annual Meeting of the World Association for Disaster and Emergency Management; May 31-June 3, 2011; Beijing, China.

Presentations at meetings (unpublished)_Jones M. Lessons learned during the Haiti earthquake. Paper presented at: 17th Annual Meeting of the World Association for Disaster and Emergency Management; May 31-June 3, 2011; Beijing, China.

Web sites - The importance of behavior in cancer prevention and early detection. American Cancer Society Web site. http://www.cancer.org/Research/ResearchProgramsFun... Behavior in Cancer PreventionandEarlyDetection/the-importance-of-behavior-in-cancer-prevention-andearly-detection. Accessed January 1, 2012. 
Online government or organization reports-World Health Organization. World Health Statistics 2011. http://www.who.int/gho/publications/world_health_statistics/EN_WHS2011_Full.pdf. Published 2011. Accessed February 6, 2012.

\section{Tables and Figures}

Only essential figures and tables should be included. Further tables, figures, photographs and appendices may be published as supplementary material with the online version on the journal. All tables and figures must be referred to in text.

Tables - submit tables either at the end of the manuscript or as separate Word or rtf files. DO NOT SUBMIT AS PDFs. Tables should be numbered in the order in which they appear in the text, using Arabic numerals. Include table title above each table. Tables should be black and white, with text in Times New Roman 12 point font. Do not use shading, and do not include spaces, tabs, or hard returns. Table footnotes should be indicated with superscript lowercase letters in alphabetical order. Tables should be no more than nine columns wide, and should fit on one printed page (portrait orientation).Tables longer or wider than a page should be split into two or more tables.

Data presented as numbers and/or percentages must add up to totals; any discrepancies must be explained in table footnotes. Numbers and corresponding percentages should be presented in the same cell, using the $\mathrm{n}(\%)$ format. Mean and standard deviation should also be presented in the same cell, using the mean (SD) format rather than the \pm format.

Figures-number all figures in the order they will appear in the text using Arabic numerals. Do not include place markers for figures in the text. Titles for figures should be included as text at the end of the manuscript, and should not be included in the figure itself. Legends should be included in the figure. Graphs, line art, diagrams, charts, and other figures should be submitted 
as black and white high resolution (300 dpi/120 pix per cm or higher) tiff files. Do Not Submit in Word Document. Color photographs and graphs may be submitted as supplemental material for online publication.

Converting images to high resolution tiff files generally requires graphics software such as PhotoShop, InDesign, or Adobe Illustrator. In some cases, it is possible to convert Word or Excel files to pdf files, then pdf files to tiff files. To convert PowerPoint files to high resolution files, see instructions at http://support.microsoft.com/default.aspx?scid=kb;... Do not submit figures as Word, PowerPoint, Excel, jpeg, or similar files. Photographs should be black and white tiff files with at least 600 dpi resolution. Please ensure that your figures are saved at final publication size (see the latest issue of the journal for column widths).

Even when your figure is saved as a 300 dpi tiff file, it may not be of sufficient resolution for print publication. If your original figure was lower resolution, simply saving it as a higher resolution file may not be helpful. Try sizing your figure to $1 / 4$ or $1 / 3$ of a page, and printing it. Is all the text clear? Is there enough contrast between bar graphs or lines and the background? White or no background is usually best. 
Permissions - illustrations or tables from other publications must be accompanied by written permission from the author and publishers of the document in which they originally appeared.

\section{Proofs and Offprints}

Page proofs will be sent to the author designated to receive correspondence. Corrections other than to printer's errors may be charged to the author. A final PDF version of the article will be supplied to the corresponding author when the article is published. Please note that no changes can be made after a paper is published online. 


\title{
5) LETTER OF ACCEPTANCE: Prehospital and Disaster Medicine
}

\section{Prehospital and Disaster Medicine}

\author{
Decision Letter Copyright \\ From: Copyright \\ To: Convrioht
}

cC:

Subject: Prehospital and Disaster Medicine - Decision on Manuscript Copyright

Body: 04-Mar-2018

Dear Dr. Alenyo:

It is a pleasure to accept the manuscript titled "A comparison between differently skilled prehospital emergency care providers in major incident triage in South Africa" for publication in Prehospital and Disaster Medicine.

The manuscript will now be placed into the Production Editing process to prepare for publication. When copy-editing is complete, you will receive electronic proof copies of the manuscript for review and final approval. The production process can take up to three months.

At this point, please upload a scan of a signed Copyright Transfer Form (designate as Copyright Transfer Form) using the following website and instructions:

https://www.cambridge.org/core/journals/prehospital-and-disastermedicine/information/transfer-copyright

ScholarOne treats the receipt of a Copyright Transfer Form as a revision. You can go to http://mc.manuscriptcentral.com/pdm?URL_MASK =c8ba0a65095a44c4842180a996900d7f to upload the file. YOUR PAPER WILL NOT BE PLACED IN QUEUE FOR PRODUCTION UNTIL THE COPYRIGHT TRANSFER FORM IS UPLOADED AS A REVISION. PLEASE DO NOT ATTACH THE COPYRIGHT TRANSFER FORM TO YOUR RESPONSE TO THIS DECISION LETTER. Once we receive final files and Copyright Transfer Form, your manuscript will be placed into Production. Due to the number of accepted manuscripts, this process can take 2-3 months to begin.

From time to time, PDM and Cambridge University Press undertake marketing efforts to highlight papers published in PDM. If you would like the PDM Editorial Board to consider your paper for this type of publicity, please fill out,sign and scan the publicity release form and upload it to ScholarOne along with your copyright transfer form.

https://www.cambridge.org/core/journais/prehospital-and-disastermedicine/information/transfer-copyright

You may also log in with your user ID and password at http://mc.manuscriptcentral.com/pdm and access your author center, where you will find your manuscript in the folder "Manuscripts With Decisions" on your author dashboard; click "create revision" and then follow the steps on the screen.

Thank you for your contribution to Prehospital and Disaster Medicine.

With regards

Copyright

Editor-in-Chief, Prehospital and Disaster Medicine

Copyright

Date Sent: 04-Mar-2018

Close Window 